\title{
Multifaceted functions of Rab23 on primary cilium and Hedgehog signaling-mediated granule cell proliferation
}

CHH Hor ${ }^{* 1,2}$, WY Leong ${ }^{2}$, and ELK Goh ${ }^{* 2,3,4,5}$

Affiliations:

${ }^{1}$ Department of Chemistry, Research Cluster on Health and Drug Discovery, Faculty of Science, Hong Kong Baptist University, Kowloon Tong, Hong Kong SAR

${ }^{2}$ Neuroscience Academic Clinical Programme, Duke-NUS Medical School, Singapore

${ }^{3}$ Department of Research, National Neuroscience Institute, Singapore

${ }^{4}$ Neuroscience and Mental Health Faculty, Lee Kong Chian School of Medicine, Nanyang Technological University, Singapore

${ }^{5}$ KK Research Center, KK Women's and Children's Hospital, Singapore

*Co-corresponding authors

Email: Eyleen_Goh@nni.com.sg; catherinehor@hkbu.edu.hk

Running title: Rab23 in hedgehog signaling and cerebellar development

Key words: Ciliary signaling, Sonic hedgehog, Primary ciliim, Rab GTPase,

Ciliogenesis, Medulloblastoma

Number of Figures: 6

\section{Conflict of Interest}

The authors declare no conflict of interest. 


\begin{abstract}
Sonic Hedgehog (Shh) signaling from the primary cilium drives cerebellar granule cell precursor (GCP) proliferation. Mutations of hedgehog $(\mathrm{Hh})$ pathway repressors could cause medulloblastoma, the most prevalent and malignant childhood brain tumor that arises from aberrant GCP proliferation. We demonstrate that brain-specific knockout of a Shh pathway repressor Rab23 in mice caused mis-patterning of cerebellar folia and elevated GCP proliferation during early development, but with no prevalent occurrence of medulloblastoma at adult stage. Strikingly, Rab23-depleted GCPs exhibited upregulated basal level of Shh pathway activities despite reduced ciliation, and were desensitized against stimulations by Shh and Smoothened (Smo) agonist in primary GCP culture. These results illustrate dual functions of Rab23 in repressing the basal level of Shh signaling, while facilitating Shh signal transduction via Shh/Smo on primary cilium. Collectively, our findings unravel instrumental roles of Rab23 in GCP proliferation and ciliogenesis. Rab23's potentiation of Shh signaling pathway through the primary cilium and Smo, suggests a potential new therapeutic for Smo/primary cilium-driven medulloblastoma.
\end{abstract}

\title{
Author Summary
}

C.H.H conceived, designed, lead, and performed all in vitro and in vivo experiments, analyzed data and wrote the manuscript. W.Y performed QPCR experiments and primary GCP cultures and analyzed data. E.L.G conceived and directed the study. 


\section{Introduction}

2

3 Cerebellar development in mammals is highly dependent on Shh signaling. In

4 particular, Shh signaling dictates the proliferation of granule cell precursors (GCP)

$5(1,2)$. GCPs give rise to granule neurons, the most abundant neuronal type in the brain.

6 In the developing cerebellum, GCPs receive mitotic signals from Shh ligands released

7 from the neighboring Purkinje cells to sustain its proliferation $(1,2)$. Besides paracrine

8 Shh signaling, GCPs were also capable of self-regulated autocrine-induced cell

9 proliferation (3). Perturbation of Shh pathway activity during early embryonic or

10 postnatal development results in cerebellar dysplasia, hypoplasia as well as malignant

11 childhood brain tumor medulloblastoma $(2,4-7)$. For example, genetic mutations of

12 Shh signaling components such as Patched (PTCH), Smoothened (SMO), Gprl61 or

$14 \quad(8-11)^{\prime}(12)$. primarily as an "antenna" on the cell membrane to receive and transduce extracellular

21 signals. In the Shh pathway, Shh ligand binds to the Ptch receptor to release its 22 suppression of Smo on the cell membrane. This subsequently triggers Smo and 23 cytosolic factors such as the Gli transcription factors and $\mathrm{SuFu}$ to interact within the 24 primary cilium before translocating into the nucleus to activate Shh downstream target

In the past decade, emergence of primary cilium as an indispensable organelle for Shh signal transduction has facilitated discoveries that recognized the seminal roles of primary cilium in cerebellar development and medulloblastoma formation. The primary cilium is a non-motile cilium found on the surface of nearly every cell. It functions genes (13-15). Although the exact molecular mechanism and trafficking cargoes that 
26 mediate dynamic ciliary entry and exit of Shh signaling components remain

27 incompletely understood, it has been well established that Shh signal transduction is

28 inevitably deregulated in the absence of a functional primary cilium. For instance,

29 knockout $(\mathrm{KO})$ of genes known to be required for primary cilium formation (i.e. Kif3a

30 or Ift88) diminished Shh activities in the cerebellum and contributed to the

31 manifestation of cerebellar hypoplasia and distorted foliation due to substantial

32 shrinkage of granule cell precursors pool $(16,17)$.

34 Intriguingly, recent findings have revealed that the primary cilium could exert both 35 inducing or suppressing forces on Shh pathway and cancer progression $(15,18,19)$.

36 Depending on the pathogenic origin of the medulloblastoma, primary cilia could potentiate tumor growth driven by Smo, and on the other hand, inhibit tumor growth driven by Gli2 (18). Adding to the complexity of the tumor biology, the same study also showed that there are ciliated and non-ciliated sub-categories of medulloblastoma, with the ones bearing primary cilia often associated with increased Shh and Wnt

41 pathway activities, whereas those without cilia do not exhibit Shh or Wnt pathway activation (18). Given the opposing functions of primary cilium on Shh pathwaymediated tumor progression, as well as the heterogeneity in ciliation capacity among

44 the tumor cells; the multifaceted functions of primary cilium might underlies variable 45 patients' responses to Smo-specific drug, Vismodegib treatment in the clinical trials 46 that targets Shh-subtype medulloblastoma $(20,21,22)$. Therefore, further insights on the 47 interaction between primary cilium and the Shh pathway, and their roles in GCP 48 proliferation would lay critical foundation for further development of effective 49 intervention for medulloblastoma. 
51 Rab23 is a brain-enriched small GTPase (21) known to antagonize the Shh pathway in

52 vivo, as evidenced by developmental mouse genetic studies. In humans, mutations of

53 RAB23 cause Carpenter syndrome, an autosomal recessive disorder characterized by

54 aberrant skull fusion, polydactyly and branchydactyly. Other variable developmental

55 abnormalities including heart defect, genu valgum, cornea defect, umbilical hernia,

56 obesity, developmental delay, as well as central nervous system (CNS)-related

57 conditions including cerebral and cerebellar malformations, hydrocephaly, intellectual

58 disability and schizophrenia (22-29). In mouse, the Rab23-encoding open brain (opb)

59 null allele mutant exhibited embryonic lethality at mid-gestation stage, exencephaly

60 and ectopic neural tube ventralization (30,31), which largely recapitulated the

61 phenotypes of other Shh repressor mutants such as Patchedl (Ptchl) and Suppressor

62 of fused (Sufu) KOs (32-34). Nonetheless, owing to the early embryonic lethality of

63 Rab23-null mutant in mouse, true implications of Rab23 in Shh signaling-mediated

64 CNS development beyond the mid-gestation stage are not known.

65

66 Genetic study revealed that Rab23 represses Hh activities via Gli2 and promotes the proteolytic cleavage of Gli3 into its cleaved repressor form (31). In addition, Rab23 also appeared to regulate Hh pathway activity through Smo. Concomittant deletion of Smo in the Rab23-null mutant has partially weakened Shh activation level in the neural tube as compared to that of Rab23 mutant (31). Besides, a molecular study in

71 mammalian cell line model reported that Rab23 mediates the protein turnover dynamics of Smo in the primary cilium, although it was not clear as to how this may influence

73 Shh pathway activity(35). Another in vitro study further revealed that Rab23

74 antagonizes the nuclear translocation of Gli1 transcription activator to impede Shh 75 pathway activation (36). Taken together, these findings suggest that Rab23 casts 
76

multiple actions in the modulation of Hh signaling cascade. However, how it orcheastrates Shh pathway in the context of GCP proliferation and medulloblastoma formation remains to be determined.

Although independent studies have implicated the functions of Rab23 in primary cilium formation and ciliary trafficking, its role in ciliogenesis remains obscure due to inconsistent observations from different cell types. For instance, overexpression of the dominant-negative form, Rab23DN perturbed ciliation in the immortalized retinal pigmented epithelial cells (37). Supporting this observation, a recent study has identified that the GDP-GTP exchange factors (GEF) of Rab23 namely Inturned and Fuzzy, were localized to the primary cilium at proximal end, and played essential role in the primary cilia formation of human and mouse cells(38). The same study demonstrated that depletion of GEF (i.e. Intu and Fuzz), or Rab23 perturbed primary cilium formation in culture IMCD3 cells. On the contrary, Rab23 $3^{--}$mouse embryo showed unaltered node cilia during early development(39). Taken together, these data suggest that Rab23's action in the primary cilium formation is possibly operating in a context-dependent manner. In the IMCD3 cells that have morphologically normal primary cilium, Rab23 forms protein complex with Kif17 and Dopamine receptor 1 (D1R), and it was required for their ciliary localization $(40,41)$. These findings indicated that Rab23 plays crucial roles in ciliary protein targeting. Despite the known function of Rab23 in primary cilium formation and Hh signaling, as well as the long perceived function of primary cilium-dependent Hh signaling in GCP proliferation, whether Rab23 is required for the primary cilium formation in the CNS and cerebellar GCP is not known. Moreover, how Rab23 may mediate primary cilium-dependent Shh 
100 signal transduction, and its impact on GCP proliferation and cerebellar development

101 remain to be further characterized.

103 In this study, we demonstrate that conditional $\mathrm{KO}$ of Rab23 in the developing mouse 104 brain at E10.5 resulted in abnormal cerebellar foliation, as well as unexpected opposing 105 changes in the cerebellar sizes and Shh activities during embryonic and postnatal 106 cerebellar development. Interestingly, our data suggest that loss of Rab23 did not casue 107 medulloblastoma despite an increase in the basal level of Shh pathway activities and 108 GCP proliferation. We found that KO of Rab23 affected ciliation in GCP, and rendered 109 the cells less responsive to pathway activation by Shh and Smo agonist. These results 110 suggest that the Rab23-KO GCPs have an attenuated response to paracrine Shh stimuli 111 from primary cilium. Taken together, we have uncovered novel functions of Rab23 in 112 GCP proliferation, acting both positively and negatively via Shh signaling. Our results 113 indicate that Rab23 represses basal level of Shh signaling pathway activities, while

114 facilitates Smo-mediated Shh pathway activation in a primary cilium-dependent 115 manner.

118 Results

120 Rab23 dictates proper cerebellar morphogenesis and development

121 In order to investigate the functions of Rab23 in central nervous system (CNS) 122 development, mouse bearing Nestin-cre (Nes) was crossed with Rab23-floxed (42) 123 homozygous mutant to achieve conditional knock-out (CKO) of Rab23 in the neural 124 progenitor cells at approximately embryonic (E) day 10.5. Gross morphological 
125 examination of the whole brain isolated from Nes-CKO mutant revealed noticeable

126 cerebellar enlargement at earlier developmental stages (i.e. postnatal (P) day one and

127 four) but appeared smaller at later adult stage as compared to the control (Rab23fff

128 counterpart (Fig. 1A-B, E, yellow asterisk). Histological examination of the mid-sagital

129 cerebellar sections by hematoxylin-eosin (H\&E) staining revealed cerebellar dysplasia

130 in Nes-CKO brains. This was consistently observed at P1, P4 and adult stages (Fig. 1C-

131 D, F). Disrupted patterning of the cerebellar folia was more prominent at the caudal

132 region. Moreover, the external granular layer (EGL) at the posterior lobules appeared

133 thicker and disorganized as compared to the control group (Fig. 1C-D, red arrows). In

134 the adult mutant, the posterior cerebellar folia were irregularly formed and lack

135 distinctive laminar layering of molecular layer (ML), Purkinje cell layer (PCL) and

136 internal granule layer (IGL) (Fig 1F). Taken together, these data indicate that a loss of

137 Rab23 resulted in defects in cerebellar folia patterning during postnatal CNS

138 development.

140 Depletion of Rab23 disrupted cerebellar radial glial scaffold and innervations of

141 granule cells

142 The disorganized laminar layering, as well as the cerebellar folia anomaly prompted

143 further examination of the Bergmann glial (Bg) scaffold, which acts as the 144 cytoarchitectural scaffold to aid in neuronal migration and lamination (43-45). We used 145 antibodies against Nestin and glial fibrillary acidic protein (GFAP) to immunolabel 146 radial glia and the Bg scaffold at early postnatal and later adult stages respectively. In

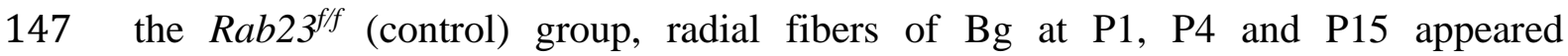
148 perpendicularly aligned and extended from the cell bodies at the lower ML and PCL 149 towards the pial surface of the cerebellum. In contrast, the processes of Nes-CKO 
mutant $\mathrm{Bg}$ in the disrupted lobules appeared tangled and misaligned, with some of them

151 unable to extend processes to the pial surface, thus indicating an impairment of the $\mathrm{Bg}$

were detectable at the pial surface in $\mathrm{P} 4$ (Fig 2B, asterisk) and adult cerebellum (Fig. 2D, white arrows). In line with this defect, the NeuN-positive granule cells in the adult mutant were aberrantly localized to the pial surface and the ML instead of the deeper IGL. In addition, a subpopulation of the granule cells was randomly scattered at the posterior region, concomitant with a loss of laminar structure. The cell soma of GFAPpositive astrocytes/Bg were also found to be ectopically misplaced at the pial surface and ML, indicating a misalignment of radial glial scaffold at the adult stage (Fig. 2D,

160 D', D"). These data indicate that an abnormal glial scaffold in Nes-CKO mutants may 161 hinder proper invagination and migration of granule cells to the deeper IGL during early postnatal cerebellar development.

164 Given the defective radial glial scaffold and ectopic accumulation of granule cells at 165 the pial surface and ML, we asked if the inward radial migration of granule cell at the earlier embryonic and postnatal stages was affected. Anti-Pax6 antibody was used to

167 immunolabel both amplifying granule cell precursors (GCP) transiently residing in the

168 EGL (source of granule cells), and the early inwardly migrated post-mitotic granule 169 cells in the granular layer at E15.5 (46). The Pax6-expressing granule cells in the 170 control group were more well-dispersed and scattered further into the deeper granule 171 layer. Conversely, Rab23-depleted granule cells appeared less dispersed and are largely 172 confined to the region adjacent to the EGL (Fig. 3A, yellow dotted lines). Because all 173 granule cells arise from the EGL and innervate the inner granular layer as they undergo 174 maturation, we quantified the innervation/migration rate by counting the number of 
175 Pax6-positive cells that have populated the inner granular layer (innervated) against total Pax6-positive cells. Indeed, the proportion of innervated granule cells in the Nes-

177 CKO mutant appeared markedly reduced compared to the control counterpart (Fig. 3B),

178 suggesting an impaired or delayed innervation. In addition, a two-hour EdU-pulse

179 labeling assay was used to track all early innervated progenitor cells. The proportion of

180 EdU-labelled cells that have innervated (from EGL - magenta arrows, and VZ - yellow

181 arrows) the granular layer was scored against all EdU-labelled cells. Similarly, Rab23-

182 depleted cells showed a lower percentage of innervated cells in the granular layer (Fig. 183 3C).

185 For the postnatal stage, we examined the migration of granule cells by 48 hours EdU186 pulse labelling for P5 to P7. The percentages of cell innervation in different lobules 187 were then analyzed by quantifying the percentages of EdU-labelled cells residing in the 188 EGL, ML and IGL of each lobule. In agreement with the results from the embryonic 189 stage (Fig. 3A-C), the proportions of mutant cells reaching IGL were greatly reduced, 190 concomitant with an increase in the percentage of cells that are accumulated in the EGL 191 (Fig. 3D-E). Taken together, these data suggest that deletion of Rab23 caused a 192 misalignment of the radial glial scaffold, leading to perturbations in granule cells 193 innervation and lamination. As a result, the cerebellar laminae and folia could not be 194 properly formed during postnatal cerebellar development.

Rab23-ablation caused thickened EGL and enhanced GCP proliferation, but no

198 In view of the thickened EGL observed in H\&E staining, as well as the enlarged 199 cerebellum of Nes-CKO at P1 and P4, a more detailed analysis of cell proliferation is 
warranted. We performed co-immunolabeling of Pax6 and Calbindin to visualized GCP

201 and Purkinje cells respectively. At P1, there was an overall increase in the number of

202 Pax6-expressing GCPs in the Nes-CKO mutant compared to the control. Besides, the

203 Pax6-labelled EGL in Nes-CKO appeared greatly thickened, more so near the posterior

204 folia (Fig. 4A, white asterisks). On the other hand, the Calbindin-expressing Purkinje

205 cells in the mutant PCL appeared to be lower in density and more sparsely distributed

206 as compared to the more densely aligned Purkinje cell layer in the control counterpart

207 (Fig. 4B), implying a perturbed PCL lamination.

Two hours EdU-pulse labelling was performed on E15.5 and P4 animals to probe GCP proliferation in further details. Compared to the control, the pools of EdU-positive

211 proliferative cells are substantially expanded in the EGL of Nes-CKO mutant at both

212 time points (Fig. 4C-D, red and yellow double heads arrows), indicating aberrantly

213 enhanced GCP proliferation during both embryonic and postnatal cerebellar

214 development. These phenotypes were further confirmed by a quantification of EdU-

215 positive nuclei in the EGL at E15.5, which revealed a significant up-regulation of 216 proliferative cells in the mutant EGL as compared to the control group (Fig. 4E).

217 Accordingly, another cell proliferation marker, Ki67, and a GCP-specific marker Atoh1,

218 also showed markedly elevated expression levels in the cerebellar tissue of Nes-CKO 219 mutants (Fig. 4F). Taken together, these data suggest that depletion of Rab23 220 potentiated GCP proliferation during early cerebellar development. Excessive GCP 221 proliferation often give rise to medulloblastoma $(47) \cdot(48,49)$. Given this, one would 222 expect the development of medulloblastoma at a later postnatal stage. However, we did 223 not find detectable manifestation of medulloblastoma in adult mutant animals, despite 
224 the occurrence of hyperplastic lesions-like tissue clumps at P4 (Fig. 1F, 2B-asterisk,

225 D-white arrows).

Shh signaling is differentially perturbed in the developing cerebellum

228 Previous genetic studies have reported that $R a b 23$ negatively regulates Shh signaling

229 (30,50). As Shh signaling is the key signaling pathway that modulates GCP 230 proliferation $(1,2,51,52)$, we reasoned that it is likely a main factor driving aberrant

231 GCP proliferation in the Rab23-deficient cerebellum. To address this possibility, we 232 examined the expressions of Gli transcription factors, which are downstream effectors 233 of Shh signaling in CNS development. The Shh signaling activities in cerebellar tissue 234 were examined in both embryonic E15.5 and late postnatal P15. In accordance with the 235 increased CGP proliferation in Nes-CKO at E15.5 and P4, Shh signaling pathway 236 activities were robustly up-regulated at E15.5, as shown by an increase in Gli1 and Gli2 237 expressions compared to the control group (Fig. 5A). Intriguingly, at P15, the 238 expression level of Glil transcripts was significantly down-regulated in the Nes-CKO 239 mutant cerebellum, despite up-regulated levels of Gli2, Gli3, Ki67 and Atohl (Fig. 5B). 240 Because Glil activates Shh-regulated genes, and its expression is dependent on both 241 Gli2 and Gli3, it could serve as the ultimate readout of Shh signaling pathway activitity 242 (C Brian Bai \& Joyner, 2001; C Brian Bai, Stephen, \& Joyner, 2004; Lee, Platt, 243 Censullo, \& Ruiz i Altaba, 1997). We also compared the expression profile of Gli1 244 transcripts at embryonic and postnatal stages. Compared to the control group which 245 exhibited relatively unaltered Glil transcript level between E15.5 and P15, the Nes246 CKO mutant showed a significant reduction in Glil transcript level at P15 compared to 247 its embryonic stage (Fig. 5C). Given the perturbed Shh signaling pathway activities, we 248 further examined if the $S h h$ transcripts were affected. Interestingly, $S h h$ transcripts level 
249 in the Nes-CKO appeared largely unchanged at both developmental time points (Fig.

250 5A-B), implying that the mutant cerebellar tissues are not short of Shh stimulants

251 despite the greatly mis-patterned cerebellum. Taken together, these data show that the

252 Shh signaling activities in the Nes-CKO mutants were initially enhanced during

253 embryonic cerebellar patterning, but became down-regulated at later postnatal time

254 point as compared to the control counterpart. Notably, this correlated well with the

255 differential changes in the cerebellar size as aforementioned (Fig. 1A-B, E). Together,

256 these results revealed that Shh signaling pathway activities were differentially

257 perturbed as a result of Rab23 deficiency during embryonic and postnatal stages of

258 cerebellar patterning.

Rab23 could regulate Shh signaling in the GCP at basal level as well as in a cilium-

\section{dependent manner}

262 The alterations of Shh signaling pathway activities on GCP in the whole cerebellar

263 tissues could be a secondary effect resulting from abnormal changes in the

264 cellular/tissue composition. We ruled out this possibility by monitoring Shh signaling

265 in primary GCP culture isolated from P7 cerebellar tissue. Primary culture data show

266 that Rab23-KO GCP indeed exhibited elevated expressions of Gli1 mRNA at the basal

267 level, indicating an over-activation of Shh pathways in the Nes-Cre mutant GCP.

268 Accordingly, primary culture of mutant GCP also displayed potentiated cell

269 proliferation, as illustrated by the up-regulation of Atohl and Ki67, as well as an

270 increase in the percentage of EdU-positive proliferative cells (Fig. 6A-C). Furthermore,

271 Shh pathway over-activation and cell proliferation were significantly inhibited by co-

272 expressing Rab23 wild-type cDNA, or its constitutive active form, $R a b 23 Q L$, in the KO

273 GCP (Fig. 6A-C), suggesting that the effects observed were indeed due to the loss-of 
274 Rab23 gene functions. Together, these results suggest a negative role for Rab23 in

275 regulating Shh signaling-mediated GCP proliferation.

277 Previous findings have demonstrated that primary cilium is required for Shh signaling278 mediated CGP proliferation during cerebellar development (16,17). Rab23 has also

279 been reported to be involved in ciliogenesis and ciliary signaling in other cell types 280 (40,41,56). Therefore, it is conceivable that the impact of Rab23 on GCP proliferation 281 was exerted through changes to the primary cilia. We examined primary cilia 282 morphology in the E15.5 and P15 cerebellar GCP by immunolabeling of Arl13b, a 283 primary cilium-specific marker. Interestingly, Arl13b immunostaining showed that the 284 Rab23-depleted GCPs exhibited a significantly reduced number of cells bearing 285 primary cilium, whereas nearly all GCPs in the control counterpart showed positive 286 staining of Arl13b (Fig. 6E). This finding is further strengthened by the analysis of 287 primary cilia in primary GCP culture isolated from P7 cerebellar tissues, whereby the 288 Rab23-deleted GCPs in culture similarly displayed a significant reduction of ciliation, 289 which could be reversed by co-expressing Rab23 wild-type cDNA, or the constitutive 290 active Rab23QL (Fig 6D). Taken together, our data provide the first indication that 291 Rab23 influences ciliogenesis in the cerebellar GCP in vivo. Importantly, these results 292 also hinted at a novel cilium-dependent role of Rab23 in coordinating Shh pathway and 293 GCP proliferation.

295 Given the perturbations in primary cilia morphology, we hypothesized that Rab23-KO 296 GCP may be compromised in primary cilium-dependent Shh signal transduction. In 297 order to address this hypothesis, primary GCP cultures were subjected to Shh ligand 298 stimulation in vitro. Despite its higher basal level of Shh signaling activities as 
compared to control (Fig. 6A), Rab23-KO GCP showed markedly weaker response to

300 Shh ligand stimulation, as illustrated by a lower fold-increase in the expression of Gli1

301 mRNA as compared to the control counterpart (Fig. 6F). Rab23-KO GCPs also

302 exhibited lower fold-enhancement in cell division as compared to the control group, in

303 which the expression of Ki67, as well as Atohl were both significantly lower than

304 control group upon Shh ligand stimulation (Fig. 6F). To check if the Shh signaling

305 occurs through Smo, GCP cultures were treated with a Smo agonist that promotes its

306 localization to the cilium. Control GCPs exhibited robust elevation Glil expression 24

307 hours after SAG treatment (Fig. 6G). Conversely, Rab23-KO GCPs' response to SAG

308 induction was significantly compromised, as shown by lower expression level of Gli1

309 (Fig. 6G), thus implying a desensitization to Shh signaling at the level of primary cilium

310 and Smo. Together, these data demonstrated that silencing Rab23 impaired ciliation

311 and GCP's response to Shh or Smo stimulations, thereby impeding Shh-mediated GCP

312 proliferation. These suggest a novel positive role of Rab23 in modulating primary

313 cilium-dependent Shh signaling and GCP expansion during early cerebellar

314 development.

\section{Discussion}

318 Fine tuning Shh signaling during cerebellar development is essential to facilitate a

319 temporally and spatially-defined transit amplification of granule cell precursors (GCP)

320 to ensure proper patterning and growth of the cerebellar folia $(2,57-59)$. We have

321 demonstrated here that $R a b 23$ has a role in the patterning and growth of cerebellar folia

322 during early cerebellar development. Deletion of Rab23 resulted in foliation anomalies

323 due to dramatically perturbed radial glial scaffold formation, granule cells lamination 
324 and GCP proliferation. This study presents Rab23 as a novel regulator of GCP

325 proliferation, remarkably, acting both positively and negatively via the Shh signaling

326 pathway. Excitingly, our data showed for the first time that Rab23 has a role in primary

327 cilium-dependent Shh signal transduction during cerebellar development. This

328 demonstration is made possible as the brain-specific KO of Rab23 in our genetic model

329 did not result in mid-gestation lethality in mice as compared to a global loss of Rab23

330 in the open brain mutant.

332 Previous examination of primary cilia in the node of 2 to 6 somite stage Rab23-null

333 embryo reported largely unaltered morphology and similar overall percentage of

334 ciliation as compared to the control (39). Interestingly, unlike the node cilia, our data

335 revealed defective ciliation in the Rab23-null GCP during embryonic and early

336 postnatal cerebellar development. siRNA-mediated knockdown studies performed on

337 different cell lines have reported inconsistent conclusions with regards to the role of

338 Rab23 in ciliogenesis $(40,41,56,60)$. These discrepancies suggest that the functions of

339 Rab23 in primary cilia could vary in a context or cell-type specific manner. Our data

340 supported a GCP-specific role of Rab23 in ciliogenesis in vivo. In line with the in vivo

341 data, primary culture of Rab23-KO GCPs also showed deficiencies in ciliation and

342 compromised response to Shh ligand and SAG-mediated Smo activation, implicating a

343 disrupted primary cilium-dependent Shh signaling.

345 Mutations of Shh pathway repressor genes, including Ptchl, Gprl61 and Sufu,

346 commonly lead to the development of medulloblastoma(9,10,61,62) via Gli1

347 upregulation (63). We showed that loss of Rab23, unlike other Shh repressors, did not

348 promote the development of medulloblastoma despite the basal level up-regulation of 
349 Glil expression in the GCPs. We further showed that the overall amount of Shh ligands

350 in Rab23-deleted cerebellar tissues remained relatively similar to that of control at both

351 embryonic and postnatal stages, suggesting a sufficient source of Shh stimulants in the

352 KO cerebellar tissue environment. Given the above, we deduce that one possible

353 explanation for an absence of tumorigenicity is the defective primary cilium in Rab23-

354 KO GCPs. The compromised response to primary cilium-dependent Shh activation may

355 lead to insufficient paracrine pathway stimulations to drive tumor formation in the

356 Rab23-KO cerebellum. This is in line with the indispensable role of primary cilium for 357 medulloblastoma formation $(18,64)$.

359 Harboring the primary cilium defect in GCP, Rab23-KO cerebellum partially 360 phenocopied other ciliopathy mutants, which often exhibit severe cerebellar size 361 shrinkage, abnormal foliation and reduced GCP proliferation due to impaired Shh 362 signaling $(16-18,64)$. In this regard, the postnatal Nes-CKO displayed profoundly mis363 patterned folia, and smaller cerebellum at later adult stage, similar to other ciliopathy 364 mutants. Nevertheless, in contrast to most ciliopathy mutants, Shh signaling in the 365 Rab23-KO mutant was not completely inhibited. Instead, there was a ligand366 independent upregulation of Shh pathway at basal level, which underlies the increase 367 in GCP proliferation and transiently enlarged cerebellum at earlier postnatal stages. 368 Rab23 was known to influence Gli2 and Gli3 expression at the transcript level (65) and 369 it could also antagonize Gli1's nuclear translocation and transcriptional activation in 370 cytosolic compartment in the absence of ligand stimulation (36). Given the ligand371 independent function of Rab23 in Shh pathway, it is therefore plausible that the Shh 372 pathway in GCP became over-activated due to a basal increase in Gli activations in the 373 absence of Rab23 function. However, limited by the incompetency to respond to Smo 
374 activation from cell/cilium membrane (Fig 6F-G), Rab23-deficeint GCP could not

375 reach or sustain the full capacity of ectopic Shh pathway activation, causing them less

376 susceptible to tumor formation as compared to other repressors such as Ptchl and Sufu

377 mutants that are not known to exhibit primary cilium defect.

379 Previous work has demonstrated that Rab23 maintains the overexpressed-Smo protein 380 turnover in the primary cilium of MDCK cells upon Shh stimulation (66), however, the 381 underlying mechanism, and how this regulation would affect Shh signaling output 382 remain elusive. Our data show that Rab23-depleted GCPs were less responsive to a 383 Smo agonist (SAG). As SAG activates Shh signaling pathway by facilitating Smo 384 translocation to the cilium axoneme, the compromised response observed in mutant 385 cells could possibly cause by the lack of intact and functional primary cilium for Smo386 mediated signaling transduction, and/or impaired maintenance of Smo turnover in the 387 primary cilium of Rab23-KO GCP. Our data suggest that the cilium malformation could 388 be the underlying reason. However, the relatively short cilia in GCP cells was 389 technically difficult for detection or quantification of the cilium localization of Smo in 390 primary GCP. We are therefore not able to ascertain if the ciliary turnover/trafficking 391 of Smo protein is affected in the mutant GCP. Nevertheless, given its previous 392 implicated role on Smo protein turnover in MDCK cells (66), Rab23 could potentially 393 mediate the Smo recycling in primary cilium to influence Shh pathway.

395 Taken together, our findings suggest that Rab23 confers dual functions in regulating 396 Shh signaling and GCP proliferation; it potentiates primary cilium and Shh/Smo397 dependent signaling cascade, while antagonizing basal level Gli transcriptional 398 activation. Our data thus present a previously unappreciated aspect of Rab23 in 
399 mediating Shh signaling upstream of Smo. This study sheds new light into the genetic

400 and mechanistic insights underpinning Shh signaling-mediated GCP proliferation and

401 cerebellar development.

\section{Materials and Methods}

Animals. Rab23-floxed animal was generated by Ozgene Pty Ltd. Conditional Rab23floxed allele was designed by flanking exon 4 for Rab23 gene with loxP sites. NestinCre (Jackson Lab cat. no. 003771) was a kind gift from Shawn Je H.S. form Duke-Nus Medical School. All animals were housed in Specific Pathogen Free (SPF) animal 409 facility at Duke-NUS Medical School, Singapore. All animal related procedures were 410 carried out in compliance to animal handling guidelines and protocol approved by 411 IACUC Singhealth, Singapore.

413 Expression vectors. For in vitro viral transduction assay, Rab23 over-expression or 414 cDNA were cloned into lentiviral pFUGW backbone. Wild-type (WT) Rab23 415 overexpression construct, previously described full-length Rab23 sequence (67) was 416 sub-cloned into pFUGW vector driven by Ubc promoter. All plasmids were amplified 417 according to the recommended protocol using Endofree ${ }^{\circledR}$ plasmid purification kit 418 (Qiagen, Germany).

419 Viral transduction and culturing of mouse primary GCP. For viral transduction of 420 primary GCP, self-inactivating murine lentiviruses were prepared according to 421 previously described protocol (42). GCP culture method was modified from standard 422 protocol. Briefly, P7 cerebellar tissues dissected were cut into small pieces and digested 423 in digestion buffer (EBSS / Papain, 1000 times dilution factor (Worthington 
424 Biochemical Corporation cat\#:3126) / $0.1 \mathrm{mg} / \mathrm{ml}$ DNaseI (Roche cat\#:11284932001) /

$4255.5 \mathrm{mM}$ cysteine-HCL) for $15 \mathrm{mins}$ at $37^{\circ} \mathrm{C}$ prior to dissociation into single cells.

426 Digestion was terminated by resuspension in $10 \% \mathrm{FBS} /$ culture medium. Suspension

427 culture was passed through $70 \mu \mathrm{m}$ cell strainer (Corning cat\#352350) to remove

428 undigested tissue clumps. Dissociated single-cell GCPs were plated on poly-D-lysine

429 (Sigma Aldrich cat\#: P6407) coated culture plates at the desired cell densities in

430 Neurobasal (Gibco®, Life Technologies, USA) medium containing B27 supplement,

431 200uM GlutaMAX ${ }^{\mathrm{TM}}-\mathrm{I}$ (Gibco®, Life Technologies, USA), sodium pyruvate $(1 \mathrm{mM})$,

432 penicilin/streptomycin and $\mathrm{KCl}(250 \mu \mathrm{M})$. Half of the culture medium was refreshed

433 every other day. Viral transduction was performed 2 to 3 hours after culture while

434 replacing fresh culture medium. The efficiencies of overexpression were validated by

435 real-time QPCR assay of DIV7 culture. For SAG stimulation, $0.2 \mu \mathrm{M}$ of SAG (Cayman

436 Chemical, cat\#: 11914-1) was added to the DIV 1 culture 24 hours prior to total RNA

437 extraction. Equal volume of DMSO was added as the untreated negative control group.

438 For Shh stimulation, $2 \mu \mathrm{g} / \mathrm{ml}$ of Shh (Stem Cell Technologies, cat\#: 78065) was added

439 to the DIV 1 culture 24 hours prior to total RNA extraction.

441 EdU-pulse labelling assays. EdU labeling assay was carried out according to the

442 manufacturer's protocol. Click-iT @ EdU Alexa Fluor ${ }^{\mathrm{TM}} 647$ Imaging Kit

443 (ThermoFisher Scientific, cat \#: C10340). For GCP culture labeling, $10 \mu \mathrm{M}$ Edu was

444 added to the culture and incubated for 3 hours before fixation. For E15.5 embryos

445 labelling, $0.25 \mathrm{mg}$ EdU was injected intraperitoneally into the pregnant mice 2 hours

446 before fixing the embryo. For postnatal animals, 25ug EdU was injected

447 subcutaneously 2 hours prior to brain fixation. 
449 Cryosectioning, immunohistochemistry and imaging. Mice were perfused with saline

450 followed by fixative in $4 \%$ paraformaldehyde (Sigma Aldrich cat\#:P6148) /

451 HistoChoice (Amresco, cat\#: H120) mixture of 1:1 ratio, and whole brains extracted

452 were post-fix at $4{ }^{\circ} \mathrm{C}$ for 2 hours, saturated in $30 \%$ sucrose in $0.12 \mathrm{M}$ phosphate buffer

453 and subjected to cryosection at $20 \mu \mathrm{m}$ thickness. All cerebellar tissues were sectioned

454 at sagital angle and mounted on pre-coated glass slides (Superfrost ${ }^{\circledR}$ Plus,

455 Fisherbrand®). Mid-sagital sections were selected for immunostaining. Antibodies and

456 the dilution factor used were: Pax6 (Covance, 1:1000), Nestin (Sigma, 1:800), NeuN

457 (Milipore, 1:800), GFAP (Milipore, 1:1000), Arl13b (Proteintech, 1:1000). For histo-

458 immunostaining, tissue sections were incubated at $100{ }^{\circ} \mathrm{C}$ for $10 \mathrm{mins}$ in $\mathrm{pH} 610 \mathrm{mM}$

459 sodium citrate buffer with $0.05 \%$ Tween- 20 for antigen retrieval, washed twice with

460 phosphate buffer saline (PBS), blocked 1 hour in $1 \% \mathrm{BSA} / 2 \%$ horse serum/ $0.3 \%$ Tx-

461100 and incubated $4^{\circ} \mathrm{C}$ overnight with primary antibodies diluted in blocking buffer.

462 After 3 times of 5 minutes washes with PBS, tissue sections were incubated with

463 secondary antibodies (Alexa Fluor®, Life Technologies, USA) for 1 hour (hr) at room

464 temperature. Tissue sections were mounted in mounting media after 3 times PBS

465 washes. Fluorescence images were taken using Zeiss LSM710 confocal system.

467 Real-time quantitative PCR. Total RNA was extracted using Qiagen's RNeasy Mini

468 Kit. Equal amount of total RNAs from each sample were subjected to reverse

469 transcription to produce cDNA. Equal volume of cDNA was used to perform

470 quantitative PCR assay using SYBR $^{\circledR}$ Select Master Mix (Applied Biosystems ${ }^{\mathrm{TM}}$

471 \#4472908). Standard QPCR protocol was carried out according to manufacturer's

472 instruction manual. Primers used were: mouse GAPDH: F-5'-

473 TTCACCACCATGGAGAAGGC-3', R-5'- GGCATGGACTGTGGTCATGA-3'; 
mouse

Rab23:

F-5'-AGGCCTACTATCGAGGAGCC-3',

R-5'-

TTAGCCTTTTGGCCAGTCCC-3';

mouse

Gli1:

F-5'-

CCCATAGGGTCTCGGGGTCTCAAAC-3',

R-5'-

GGAGGACCTGCGGCTGACTGTGTA

A-3'; mouse Gli2: F-5'-CATGGTATCCCTAGCTCCTC-3', $\quad$ R-5'-

479 GATGGCATCAAAGTCAATCT-3'; mouse

Gli3:

F-5'-

CATGAACAGCCCTTTAAGAC-3', R-5'-TGATATGTGAGGTAGCACCA-3';

Ptch1:

F-5'-TGCTGTGCCTGTGGTCATCCTGATT-3',

R-5'TTGGTATCTTGACCTTCCCCATCAG-3’.

\section{Acknowledgement}

We sincerely thank C.C. Hui and B.L Tang for sharing critical and constructive comments and suggestions for the successful completion of this project. This study was supported by the National Medical Research Council - Young Individual Research

491 Grant (NMRC/OFYIRG/0079/2018), HKBU Tier 1 and Tier 2 Start-up Grant (RG492 SGT2/18-19/SCI/009) to C.H.H; the National Medical Research Council 493 Collaborative Research Grant (NMRC/CBRG/0094/2015) and Ministry of Education 494 (MOE) Tier 2 grant (MOE2015-T2-1-022) and Tier 3 grant (MOE2017-T3-1-002) to 495 E.L.G. 
Figure 1. Nestin-Cre-driven knock-out of $\operatorname{Rab23}$ causes expanded cerebellar size and abnormal foliation.

502 A) Representative whole mount images of control and Nes-CKO mutant brains 503 showing gross morphology of mouse cerebellum at P1, P4 and P15.

504 B) P4 cerebellar sizes as determined by measuring 2D surface area of cerebellum on 505 images captured at similar angle. Control, $\mathrm{n}=6 ; \mathrm{CKO}, \mathrm{n}=4$ Statistical significance, 506 unpaired student t-test. P value $* * \leq 0.01$. Error bars depict $\pm \mathrm{SE}$

507 C-D) Representative images showing H\&E staining of control and Nes-CKO cerebellar 508 sagital sections of P1 (C) and P4 (D) animals. Red arrows highlight morphological 509 changes in the external granule layer of Nes-CKO compared to the control.

510 E) Representative image showing whole brain of 2 months adult mice. Yellow asterisk

511 shows smaller cerebellum of Nes-CKO mutant compared to the control.

512 F) Representative images showing H\&E staining of sagital cerebellar sections of 10 513 months adult mice.

517 A-B) Representative images showing immunostaining of Nestin (red) on P1 (A) and P4

518 (B) sagital cerebellar tissue sections to illustrate radial glial scaffold.

519 C) Representative images showing immunostaining of GFAP (red) on P15 sagital 520 cerebellar tissue sections to illustrate radial glial scaffold.

521 D) Representative images showing co-immunostaining of NeuN and GFAP on ten 522 months adult cerebellum to illustrate cerebellar cytoarchitecture, laminar layers and 523 glial cells. 
524 D'-D”) Close up images showing NeuN-positive granule neurons and GFAP-positive 525 glial cells at the internal granule layer and pial surface.

Figure 3. Depletion of Rab23 leads to GCP migration defect

528 A) Representative images showing immunostaining of Pax6 on E15.5 sagital sections

529 of cerebellar primordium to illustrate the GCPs residing in the EGL and early inward 530 migrating GCPs. White arrows show inward migration paths. EGL, external granular 531 layer; RL, rhombic lip

532 B) Quantification of the proportion of innervated Pax6+ GCPs against all Pax6-labelled 533 GCPs. 2 to 3 sections $(\sim 50-100 \mu \mathrm{m}$ apart $)$ of the cerebellar primordium were counted 534 for each animal. Control, $\mathrm{n}=3 ; \mathrm{CKO}, \mathrm{n}=3$. Statistical significance, unpaired student $535 \quad$ t-test. $\mathrm{P}$ value $* \leq 0.05$. Error bars depict $\pm \mathrm{SE}$

536 C) Representative image and graph showing two-hours EdU labelled progenitors in the 537 cerebellar primordium. Magenta arrows show migration paths of progenitors from 538 EGL, yellow arrows show migration paths of progenitors from VL. Control, $\mathrm{n}=3$; $539 \mathrm{CKO}, \mathrm{n}=3.2$ to 3 sections $(\sim 50-100 \mu \mathrm{m}$ apart $)$ were counted for each animal. Statistical 540 significance, unpaired student t-test. $\mathrm{P}$ value $* * * \leq 0.001$. Error bars depict $\pm \mathrm{SE}$

541 D) Representative images showing cerebellar lobules of 48 hours Edu-labelled cells 542 from P5-P7 to illustrate proportions of cells innervated the IGL after 48 hours of pulse543 chase labeling. EGL, external granular layer; ML, molecular layer; ICL, internal 544 granule layer.

545 E) Quantification of the proportion of Edu-labelled cells in each laminar layer as 546 indicated. Control, $\mathrm{n}=3 ; \mathrm{CKO}, \mathrm{n}=3$. Statistical significance, two-way ANOVA, 547 Bonferroni posttests. P value $* * * \leq 0.001, * * \leq 0.01, * \leq 0.05$. Error bars depict $\pm \mathrm{SE}$ 
551 A-B) Representative images showing co-immunostaining of Pax6 (green) and

Calbindin (red) on P1 sagital sections of cerebellum to illustrate the GCPs and Purkinje cells layers. Asterisks show a thickened EGL layer in Nes-CKO cerebellum compared

554 to the control.

555 C-D) Representative images showing two-hours EdU labelled dividing progenitors in the E15.5 (C) and P4 (D) cerebellum. Double headed arrows highlight expanded pools

557 of dividing cells in the Nes-CKO EGL as compared to the control counterparts.

558 E) Quantification of the percentages of 2 hours Edu-labelled proliferative cells in the 559 EGL at E15.5. 2 sections $(\sim 100 \mu \mathrm{m}$ apart $)$ of the cerebellar primordium were counted 560 for each animal. Control, $\mathrm{n}=3 ; \mathrm{CKO} \mathrm{n}=3$. Statistical significance, unpaired student $\mathrm{t}-$ 561 test. $\mathrm{P}$ value $* * * \leq 0.001$. Error bars depict $\pm \mathrm{SE}$

562 F) Graphs illustrating the fold change of the gene expression levels of E15.5 cerebellar 563 tissues quantified by real-time quantitative PCR. Control, $\mathrm{n}=4 ; \mathrm{CKO} \mathrm{n}=4$. Nes-CKO 564 values were normalized to its respective control group. Statistical significance, unpaired 565 student t-test. $\mathrm{P}$ value $* * * \leq 0.001$. Error bars depict $\pm \mathrm{SE}$

567 Figure 5. Shh activity is differentially perturbed in the embryonic and postnatal 568 cerebellar tissues.

569 A) Graphs illustrating the fold change of the gene expression levels of E15.5 cerebellar 570 tissues quantified by real-time quantitative PCR. Control, $n=4 ; \mathrm{CKO} n=4$. Nes-CKO 571 values were normalized to its respective control group. Statistical significance, unpaired student t-test. $\mathrm{P}$ value $* * \leq 0.01$. Error bars depict \pm SE. n.s., not significant. 
573 B) Graphs illustrating the fold change of the gene expression levels of P15 cerebellar

574 tissues quantified by real-time quantitative PCR. Nes-CKO values were normalized to

575 its respective control group. Control, $\mathrm{n}=3$; CKO $\mathrm{n}=4$. Statistical significance,

576 unpaired student t-test. P value $* * * \leq 0.001$. Error bars depict \pm SE. n.s., not significant.

577 C) Graphs illustrating the basal level Gli1 expression profiles of E15.5 and P15

578 cerebellar tissues quantified by real-time quantitative PCR. E15.5 Control, $n=4$; CKO

$579 \mathrm{n}=4 ; \mathrm{P} 15$ Control, $\mathrm{n}=3 ; \mathrm{CKO} \mathrm{n}=4$. Statistical significance, one-way AVONA,

580 Bonferroni’s Multiple Comparison Test. P value *** $\leq 0.0001$. Error bars depict \pm SE.

581 n.s., not significant.

Figure 6. $R a b 23$ regulates ciliogenesis and Shh signaling in the GCPs.

584 A) Graphs showing gene expression levels of P7 GCPs primary cultures harvested at

585 DIV 7. Lentiviral carrying the over-expression constructs as indicated were transduced 586 into the primary cultures at Day 0, 2 to 3 hours after seeding cells. Quantifications 587 depict 4 independent experiments. Statistical significance, one-way AVONA, 588 Bonferroni's Multiple Comparison Test. P value $* * * \leq 0.0001 ; * * \leq 0.01, * \leq 0.05$. Error 589 bars depict \pm SE.

590 B-C) Representative images (B) and graph (C) showing three-hours EdU labelled 591 (magenta; blue: DAPI) dividing progenitors in P7 GCPs primary cultures of each 592 indicated groups fixed at DIV 7. Cell proliferation was determined by the percentages 593 of Edu-labelled cells out of total number of DAP-positive nuclei in each image taken. 594 For quantification of each batch, 3 fluorescence images were randomly taken from each 595 respective group as indicated. Quantifications depict 3 independent experiments. 596 Statistical significance, one-way AVONA, Bonferroni's Multiple Comparison Test. P 597 value $* * * \leq 0.0001 ; * * \leq 0.01$. Error bars depict \pm SE. 
598 D) Quantification of the percentages of ciliation in P7 GCPs primary cultures at DIV7

599 determined by counting the number of cells bearing Arl13B-labelled primary cilium

600 against all DAPI-positive nuclei in each image taken. For quantification of each batch,

6013 fluorescence images were randomly taken from each respective group as indicated.

602 Quantifications depict 3 independent experiments. Statistical significance, one-way

603 AVONA, Bonferroni’s Multiple Comparison Test. P value $* * * \leq 0.0001 ; * * \leq 0.01$.

604 Error bars depict \pm SE.

605 E) Representative images showing immunostaining of Arl13B on E15.5 (top) and P15

606 (bottom) sagital sections to illustrate the primary cilia of GCPs residing in the EGL.

607 EGL, external granular layer; ML, molecular layer.

608 F-G) Graphs showing gene expression levels of P7 GCPs primary cultures treated with

609 Shh (F) and SAG (G) on DIV 1 respectively. Total RNAs were extracted from DIV 2

610 culture, 24 hours after the respective treatments. Quantifications depict double delta $\mathrm{Ct}$

611 values of 3 independent experiments. Delta $\mathrm{Ct}$ values of the treated groups were

612 normalized to its respective untreated group, which gave double delta $\mathrm{Ct}$ values as

613 plotted. Statistical significance, unpaired student t-test. P value $* * * \leq 0.0001 ; * * \leq 0.01$.

614 Error bars depict \pm SE. 
615

616

617

618

619

620

621

622

623

624

625

626

627

628

629

630

631

632

633

634

635

636

637

638

639

640

641

642

643

644

645

646

647

648

649

650

651

652

653

654

655

656

657

658

659

660

661

662

663

664

\section{References}

1. Wallace VA. Purkinje-cell-derived Sonic hedgehog regulates granule neuron precursor cell proliferation in the developing mouse cerebellum [Internet]. Vol. 9, Current Biology. Elsevier; 1999. p. 445-8. Available from: http://www.cell.com/current-biology/abstract/S0960-9822(99)80195-X

2. Wechsler-Reya RJ, Scott MP. Control of neuronal precursor proliferation in the cerebellum by sonic hedgehog. Neuron. 1999;22(1):103-14.

3. Gao W-Q, Heintz N, Hatten ME. Cerebellar granule cell neurogenesis is regulated by cell-cell interactions in vitro. Neuron [Internet]. 1991 May;6(5):705-15. Available from: http://linkinghub.elsevier.com/retrieve/pii/089662739190168Y

4. Basson MA, Wingate RJ. Congenital hypoplasia of the cerebellum: developmental causes and behavioral consequences. Front Neuroanat [Internet]. 2013 Sep 3 [cited 2018 Nov 23];7:29. Available from: http://www.ncbi.nlm.nih.gov/pubmed/24027500

5. Roussel MF, Hatten ME. Cerebellum development and medulloblastoma. Curr Top Dev Biol [Internet]. 2011;94:235-82. Available from: http://linkinghub.elsevier.com/retrieve/pii/B9780123809162000085

6. Dahmane N, Ruiz i Altaba A. Sonic hedgehog regulates the growth and patterning of the cerebellum. Development [Internet]. 1999 Jun;126(14):3089100. Available from: http://www.ncbi.nlm.nih.gov/pubmed/10375501

7. Lewis PM, Gritli-Linde A, Smeyne R, Kottmann A, McMahon AP. Sonic hedgehog signaling is required for expansion of granule neuron precursors and patterning of the mouse cerebellum. Dev Biol [Internet]. 2004 Jun;270(2):393410. Available from:

8. Dey J, Ditzler S, Knoblaugh SE, Hatton BA, Schelter JM, Cleary MA, et al. A Distinct Smoothened Mutation Causes Severe Cerebellar Developmental Defects and Medulloblastoma in a Novel Transgenic Mouse Model. Mol Cell Biol [Internet]. 2012;32(20):4104-15. Available from: http://mcb.asm.org/cgi/doi/10.1128/MCB.00862-12

9. Dong J, Gailani MR, Pomeroy SL, Reardon D, Bale AE. Identification ofPATCHED mutations in medulloblastomas by direct sequencing [Internet]. Vol. 16, Hum. Mutat. 2000. p. 89-90. Available from: http://doi.wiley.com/10.1002/10981004\%28200007\%2916\%3A1\%3C89\%3A\%3AAIDHUMU18\%3E3.0.CO\%3B2-7

10. Taylor MD, Liu L, Raffel C, Hui C, Mainprize TG, Zhang X, et al. Mutations in SUFU predispose to medulloblastoma. Nat Genet [Internet]. $2002 \mathrm{Jul}$ 17;31(3):306-10. Available from: http://www.nature.com/articles/ng916z

11. Raffel C, Jenkins RB, Frederick L, Hebrink D, Alderete B, Fults DW, et al. Sporadic medulloblastomas contain PTCH mutations. Cancer Res [Internet]. 1997 Mar 1;57(5):842-5. Available from: http://www.ncbi.nlm.nih.gov/pubmed/9041183

12. Begemann M, Waszak SM, Robinson GW, Jäger N, Sharma T, Knopp C, et al. Germline GPR161 mutations predispose to pediatric medulloblastoma. J Clin Oncol [Internet]. 2020 Jan 1 [cited 2020 Mar 9];38(1):43-50. Available from: http://ascopubs.org/doi/10.1200/JCO.19.00577

13. Tukachinsky H, Lopez L V, Salic A. A mechanism for vertebrate Hedgehog signaling: recruitment to cilia and dissociation of $\mathrm{SuFu}-\mathrm{Gli}$ protein complexes 
665

666

667

668

669

670

671

672

673

674

675

676

677

678

679

680

681

682

683

684

685

686

687

688

689

690

691

692

693

694

695

696

697

698

699

700

701

702

703

704

705

706

707

708

709

710

711

712

713

714
[Internet]. Vol. 191, The Journal of Cell Biology. 2010. p. 415-28. Available from: http://jcb-dataviewer.rupress.org/jcb/doi/10.1083/jcb.201004108

14. Haycraft CJ, Banizs B, Aydin-Son Y, Zhang Q, Michaud EJ, Yoder BK. Gli2 and Gli3 localize to cilia and require the intraflagellar transport protein polaris for processing and function. [Internet]. Vol. 1, PLoS Genet. 2005. p. e53. Available from:

http://eutils.ncbi.nlm.nih.gov/entrez/eutils/elink.fcgi?dbfrom=pubmed\&amp;id $=16254602 \&$ amp;retmode $=$ ref\&amp;cmd=prlinks

15. Wong SY, Seol AD, So P-L, Ermilov AN, Bichakjian CK, Epstein EH, et al. Primary cilia can both mediate and suppress Hedgehog pathway-dependent tumorigenesis. Nat Med [Internet]. 2009 Sep 23;15(9):1055-61. Available from: http://www.nature.com/articles/nm.2011

16. Spassky N, Han Y-G, Aguilar A, Strehl L, Besse L, Laclef C, et al. Primary cilia are required for cerebellar development and Shh-dependent expansion of progenitor pool. Dev Biol [Internet]. 2008 May;317(1):246-59. Available from: http://linkinghub.elsevier.com/retrieve/pii/S0012160608001395

17. Chizhikov V V., Davenport J, Zhang Q, Shih EK, Cabello OA, Fuchs JL, et al. Cilia proteins control cerebellar morphogenesis by promoting expansion of the granule progenitor pool. J Neurosci [Internet]. 2007 Sep 5;27(36):9780-9.

Available from: http://www.ncbi.nlm.nih.gov/pubmed/17804638

18. Han YG, Kim HJ, Dlugosz AA, Ellison DW, Gilbertson RJ, Alvarez-Buylla A. Dual and opposing roles of primary cilia in medulloblastoma development. Nat Med [Internet]. 2009;15(9):1062-5. Available from:

http://dx.doi.org/10.1038/nm.2020

19. Bay SN, Long AB, Caspary T. Disruption of the ciliary GTPase Arl13b suppresses Sonic hedgehog overactivation and inhibits medulloblastoma formation. Proc Natl Acad Sci [Internet]. 2018 Feb 13 [cited 2019 Jan 5];115(7):1570-5. Available from: www.pnas.org/cgi/doi/10.1073/pnas.1706977115

20. Gajjar A, Stewart CF, Ellison DW, Kaste S, Kun LE, Packer RJ, et al. Phase I Study of Vismodegib in Children with Recurrent or Refractory Medulloblastoma: A Pediatric Brain Tumor Consortium Study. Clin Cancer Res [Internet]. 2013 Nov 15 [cited 2018 Nov 28];19(22):6305-12. Available from: http://www.ncbi.nlm.nih.gov/pubmed/24077351

21. Guo A, Wang T, Ee LN, Aulia S, Kooi HC, Teng FYH, et al. Open brain gene product Rab23: Expression pattern in the adult mouse brain and functional characterization. J Neurosci Res. 2006;

22. Alessandri J-L, Dagoneau N, Laville J-M, Baruteau J, Hébert J-C, Cormier-

Daire V. RAB23 mutation in a large family from Comoros Islands with Carpenter syndrome. [Internet]. Vol. 152A, Am. J. Med. Genet. 2010. p. 9826. Available from:

http://eutils.ncbi.nlm.nih.gov/entrez/eutils/elink.fcgi?dbfrom=pubmed\&amp;id $=20358613 \&$ amp;retmode=ref\&amp;cmd=prlinks

23. Jenkins D, Seelow D, Jehee FS, Perlyn CA, Alonso LG, Bueno DF, et al. RAB23 mutations in Carpenter syndrome imply an unexpected role for hedgehog signaling in cranial-suture development and obesity. [Internet]. Vol. 80, Am. J. Hum. Genet. 2007. p. 1162-70. Available from: http://eutils.ncbi.nlm.nih.gov/entrez/eutils/elink.fcgi?dbfrom=pubmed\&amp;id $=17503333 \&$ amp;retmode $=$ ref \&amp; $\mathrm{cmd}=$ prlinks

24. Haye D, Collet C, Sembely-Taveau C, Haddad G, Denis C, Soulé N, et al. 
Prenatal findings in carpenter syndrome and a novel mutation in RAB23.

[Internet]. Vol. 164A, Am. J. Med. Genet. 2014. p. 2926-30. Available from: http://eutils.ncbi.nlm.nih.gov/entrez/eutils/elink.fcgi?dbfrom=pubmed\&amp;id $=25168863 \&$ amp;retmode $=$ ref\&amp;cmd=prlinks

25. Tarhan E, Oğuz H, Şafak MA, Samim E. The Carpenter syndrome phenotype. Int J Pediatr Otorhinolaryngol. 2004;68(3):353-7.

26. Balci S, Onol B, Eryilmaz M, Haytoglu T. A case of Carpenter syndrome diagnosed in a 20-week-old fetus with postmortem examination. Clin Genet. 1997;51(6):412-6.

27. Bersani G, Maddalena F, Pasquini M, Orlandi V, Pancheri P. Association of schizophrenia and Carpenter syndrome. Acta Neuropsychiatr [Internet]. 2003 Oct 24;15(05):304-5. Available from: https://www.cambridge.org/core/product/identifier/S0924270800004373/type/j ournal_article

28. Ivaniutsin U, Chen Y, Mason JO, Price DJ, Pratt T. Adenomatous polyposis coli is required for early events in the normal growth and differentiation of the developing cerebral cortex. [Internet]. Vol. 4, Neural Dev. 2009. p. 3. Available from:

http://eutils.ncbi.nlm.nih.gov/entrez/eutils/elink.fcgi?dbfrom=pubmed\&amp;id =19149881\&amp;retmode=ref\&amp;cmd=prlinks

29. Taravath S, Tonsgard JH. Cerebral malformations in Carpenter syndrome. Pediatr Neurol [Internet]. 1993 May 1 [cited 2018 Nov 28];9(3):230-4. Available from: http://www.ncbi.nlm.nih.gov/pubmed/8352858

30. Eggenschwiler JT, Espinoza E, Anderson K V. Rab23 is an essential negative regulator of the mouse Sonic hedgehog signalling pathway. [Internet]. Vol. 412, Nature. 2001. p. 194-8. Available from: http://eutils.ncbi.nlm.nih.gov/entrez/eutils/elink.fcgi?dbfrom=pubmed\&amp;id $=11449277 \&$ amp;retmode $=$ ref \&amp;cmd=prlinks

31. Eggenschwiler JT, Bulgakov O V, Qin J, Li T, Anderson K V. Mouse Rab23 regulates Hedgehog signaling from Smoothened to Gli proteins [Internet]. Vol. 290, Developmental Biology. 2006. p. 1-12. Available from: http://linkinghub.elsevier.com/retrieve/pii/S0012160605006299

32. Svärd J, Heby-Henricson K, Henricson KH, Persson-Lek M, Rozell B, Lauth $\mathrm{M}$, et al. Genetic elimination of Suppressor of fused reveals an essential repressor function in the mammalian Hedgehog signaling pathway. [Internet]. Vol. 10, Developmental Cell. 2006. p. 187-97. Available from: http://www.ncbi.nlm.nih.gov/sites/entrez?Db=pubmed\&amp;Cmd=ShowDetail View\&amp;TermToSearch=16459298\&amp;ordinalpos=3\&amp;itool=EntrezS ystem2.PEntrez.Pubmed.Pubmed_ResultsPanel.Pubmed_RVDocSum

33. Cooper AF. Cardiac and CNS defects in a mouse with targeted disruption of suppressor of fused [Internet]. Vol. 132, Development. 2005. p. 4407-17. Available from: http://dev.biologists.org/cgi/doi/10.1242/dev.02021

34. Jeong J. Growth and pattern of the mammalian neural tube are governed by partially overlapping feedback activities of the hedgehog antagonists patched 1 and Hhip1. Development [Internet]. 2004 Dec 2;132(1):143-54. Available from: http://dev.biologists.org/cgi/doi/10.1242/dev.01566

35. Boehlke C, Bashkurov M, Buescher A, Krick T, John A-K, Nitschke R, et al. Differential role of Rab proteins in ciliary trafficking: Rab23 regulates smoothened levels. J Cell Sci [Internet]. 2010 May 1 [cited 2018 Nov 22];123(Pt 9):1460-7. Available from: 
http://www.ncbi.nlm.nih.gov/pubmed/20375059

36. Chi S, Xie G, Liu H, Chen K, Zhang X, Li C, et al. Rab23 negatively regulates Gli1 transcriptional factor in a $\mathrm{Su}(\mathrm{Fu})$-dependent manner. Cell Signal [Internet]. 2012 Jun;24(6):1222-8. Available from: https://linkinghub.elsevier.com/retrieve/pii/S0898656812000599

37. Yoshimura S i, Egerer J, Fuchs E, Haas AK, Barr FA. Functional dissection of Rab GTPases involved in primary cilium formation [Internet]. Vol. 178, The Journal of Cell Biology. 2007. p. 363-9. Available from: http://www.jcb.org/cgi/doi/10.1083/jcb.200703047

38. Gerondopoulos A, Strutt H, Stevenson NL, Sobajima T, Levine TP, Stephens DJ, et al. Planar Cell Polarity Effector Proteins Inturned and Fuzzy Form a Rab23 GEF Complex. Curr Biol [Internet]. 2019 Oct;29(19):3323-3330.e8. Available from: https://linkinghub.elsevier.com/retrieve/pii/S0960982219310164

39. Fuller K, O\&apos;Connell JT, Gordon J, Mauti O, Eggenschwiler J. Rab23 regulates Nodal signaling in vertebrate left-right patterning independently of the Hedgehog pathway. [Internet]. Vol. 391, Developmental Biology. 2014. p. 182-95. Available from:

http://eutils.ncbi.nlm.nih.gov/entrez/eutils/elink.fcgi?dbfrom=pubmed\&amp;id $=24780629 \&$ amp;retmode $=$ ref \&amp; $c$ md $=$ prlinks

40. Lim YS, Tang BL. A role for Rab23 in the trafficking of Kif17 to the primary cilium. [Internet]. Vol. 128, J. Cell. Sci. 2015. p. 2996-3008. Available from: http://eutils.ncbi.nlm.nih.gov/entrez/eutils/elink.fcgi?dbfrom=pubmed\&amp;id $=26136363 \&$ amp;retmode=ref\&amp;cmd=prlinks

41. Leaf A, Von Zastrow M. Dopamine receptors reveal an essential role of IFT-B, KIF17, and Rab23 in delivering specific receptors to primary cilia. [Internet]. Vol. 4, Elife. 2015. Available from: http://eutils.ncbi.nlm.nih.gov/entrez/eutils/elink.fcgi?dbfrom=pubmed\&amp;id $=26182404 \&$ amp;retmode=ref\&amp; cmd=prlinks

42. Hor CHH, Goh ELK. Rab23 Regulates Radial Migration of Projection Neurons via N-cadherin. Cereb Cortex [Internet]. 2018 Apr 1;28(4):1516-31. Available from: https://academic.oup.com/cercor/article/28/4/1516/4840630

43. Xu H, Yang Y, Tang X, Zhao M, Liang F, Xu P, et al. Bergmann Glia Function in Granule Cell Migration During Cerebellum Development. Mol Neurobiol [Internet]. 2013 Apr 19 [cited 2018 Nov 16];47(2):833-44. Available from: https://link-springercom.libproxy1.nus.edu.sg/content/pdf/10.1007\%2Fs12035-013-8405-y.pdf

44. Sidman RL, Rakic P. Neuronal migration, with special reference to developing human brain: a review. Brain Res [Internet]. 1973 Nov;62(1):1-35. Available from: http://linkinghub.elsevier.com/retrieve/pii/0006899373906173

45. Yue Q. PTEN deletion in Bergmann glia leads to premature differentiation and affects laminar organization. Development [Internet]. 2005;132(14):3281-91. Available from: http://dev.biologists.org/cgi/doi/10.1242/dev.01891

46. Chung S, Kim C, Jung Y, Lee N, Jeong Y. Early cerebellar granule cell migration in the mouse embryonic development. Cell. 2010;86-95.

47. Kim JYH, Nelson AL, Algon SA, Graves O, Sturla LM, Goumnerova LC, et al. Medulloblastoma tumorigenesis diverges from cerebellar granule cell differentiation in patched heterozygous mice. Dev Biol [Internet]. 2003 Nov;263(1):50-66. Available from: http://linkinghub.elsevier.com/retrieve/pii/S0012160603004342 
48. Yoshioka K. Control of granule cell precursor proliferation in the developing cerebellum and in medulloblastoma. Biomedical Reviews. 2005.

49. Roussel MF, Hatten ME. Cerebellum development and medulloblastoma. Curr Top Dev Biol [Internet]. 2011;94:235-82. Available from: http://www.ncbi.nlm.nih.gov/pubmed/21295689

50. Evans TM, Simpson F, Parton RG, Wicking C. Characterization of Rab23, a Negative Regulator of Sonic Hedgehog Signaling. In: Methods in enzymology [Internet]. 2005. p. 759-77. Available from: http://www.ncbi.nlm.nih.gov/pubmed/16473637

51. Corrales JD. Spatial pattern of sonic hedgehog signaling through Gli genes during cerebellum development. Development [Internet]. 2004;131(22):558190. Available from: http://dev.biologists.org/cgi/doi/10.1242/dev.01438

52. Lewis PM, Gritli-Linde A, Smeyne R, Kottmann A, McMahon AP. Sonic hedgehog signaling is required for expansion of granule neuron precursors and patterning of the mouse cerebellum. [Internet]. Vol. 270, Developmental Biology. 2004. p. 393-410. Available from:

http://eutils.ncbi.nlm.nih.gov/entrez/eutils/elink.fcgi?dbfrom=pubmed\&amp;id $=15183722 \&$ amp;retmode $=$ ref\&amp;cmd=prlinks

53. Lee J, Platt KA, Censullo P, Ruiz i Altaba A. Gli1 is a target of Sonic hedgehog that induces ventral neural tube development. Development [Internet]. 1997 Jul;124(13):2537-52. Available from: http://www.ncbi.nlm.nih.gov/pubmed/9216996

54. Bai CB, Joyner AL. Gli1 can rescue the in vivo function of Gli2. Development [Internet]. 2001 Dec;128(24):5161-72. Available from:

http://www.ncbi.nlm.nih.gov/pubmed/11748151

55. Bai CB, Stephen D, Joyner AL. All mouse ventral spinal cord patterning by hedgehog is Gli dependent and involves an activator function of Gli3. Dev Cell [Internet]. 2004 Jan;6(1):103-15. Available from: http://linkinghub.elsevier.com/retrieve/pii/S1534580703003940

56. Yoshimura S, Egerer J, Fuchs E, Haas AK, Barr FA. Functional dissection of Rab GTPases involved in primary cilium formation. J Cell Biol [Internet]. 2007 Jul 30;178(3):363-9. Available from: http://www.jcb.org/lookup/doi/10.1083/jcb.200703047

57. Butts T, Green MJ, Wingate RJT. Development of the cerebellum: simple steps to make a "little brain." Development [Internet]. 2014;141(21):4031-41. Available from: http://dev.biologists.org/cgi/doi/10.1242/dev.106559

58. Wallace VA. Purkinje-cell-derived Sonic hedgehog regulates granule neuron precursor cell proliferation in the developing mouse cerebellum. Curr Biol [Internet]. 1999 Apr 22;9(8):445-8. Available from: http://linkinghub.elsevier.com/retrieve/pii/S096098229980195X

59. Miyazawa K, Himi T, Garcia V, Yamagishi H, Sato S, Ishizaki Y. A role for p27/Kip1 in the control of cerebellar granule cell precursor proliferation. J Neurosci. 2000;20(15):5756-63.

60. Hor CHH, Tang BL, Goh ELK. Rab23 and developmental disorders. Rev Neurosci [Internet]. 2018 Nov 27;29(8):849-60. Available from: http://www.degruyter.com/view/j/revneuro.2018.29.issue-8/revneuro-20170110/revneuro-2017-0110.xml

61. Begemann M, Waszak SM, Robinson GW, Jäger N, Sharma T, Knopp C, et al. Germline GPR161 mutations predispose to pediatric medulloblastoma. J Clin Oncol. 2020 Jan;38(1):43-50. 
62. Zurawel RH, Allen C, Wechsler-Reya R, Scott MP, Raffel C. Evidence that haploinsufficiency of Ptch leads to medulloblastoma in mice. Genes,

\section{Chromosom Cancer. 2000 May 1;28(1):77-81.} 63. Kimura H, Stephen D, Joyner A, Curran T. Gli1 is important for
medulloblastoma formation in Ptc1+/- mice. Oncogene [Internet]. 2005 Jun

4;24(25):4026-36. Available from: http://www.nature.com/articles/120856
64. Barakat MT, Humke EW, Scott MP. Kif3a is necessary for initiation and

65. Eggenschwiler JT, Bulgakov O V., Qin J, Li T, Anderson K V. Mouse Rab23 regulates Hedgehog signaling from Smoothened to Gli proteins. Dev Biol [Internet]. 2006 Feb 1 [cited 2018 Nov 21];290(1):1-12. Available from: https://www.sciencedirect.com/science/article/pii/S0012160605006299?via\%3

66. Boehlke C, Bashkurov M, Buescher A, Krick T, John A-K, Nitschke R, et al. 
bioRxiv preprint doi: https://doi.org/10.1101/2020.08.01.231985; this version posted August 1, 2020. The copyright holder for this preprint

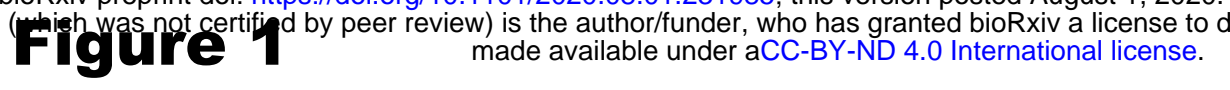

A
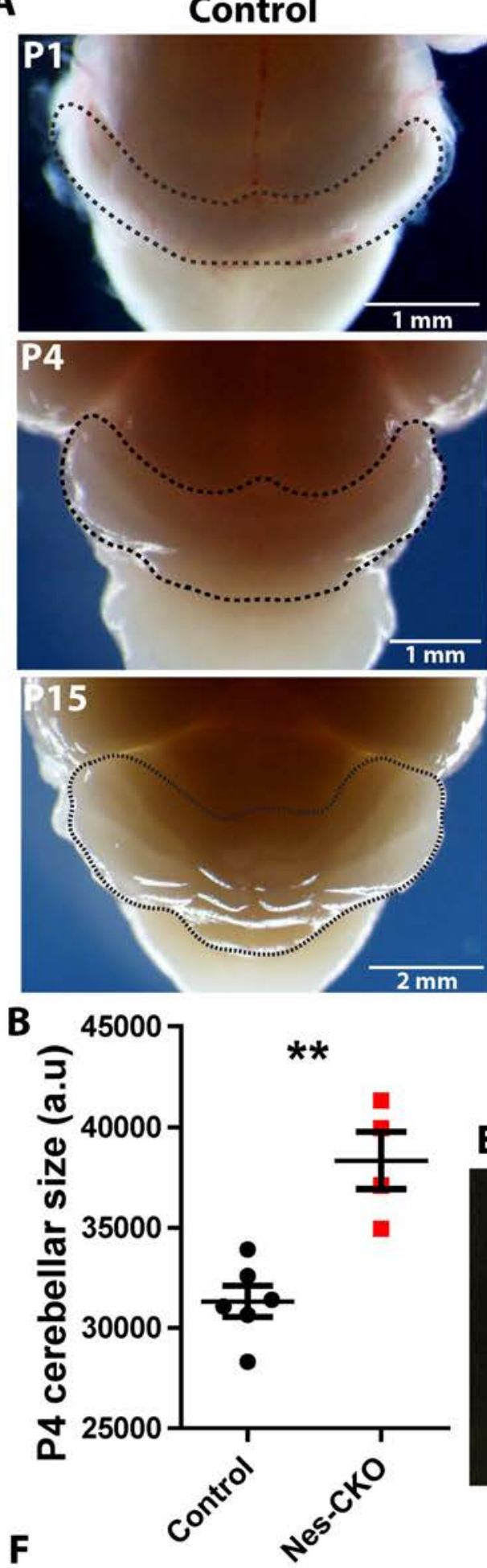

10 months

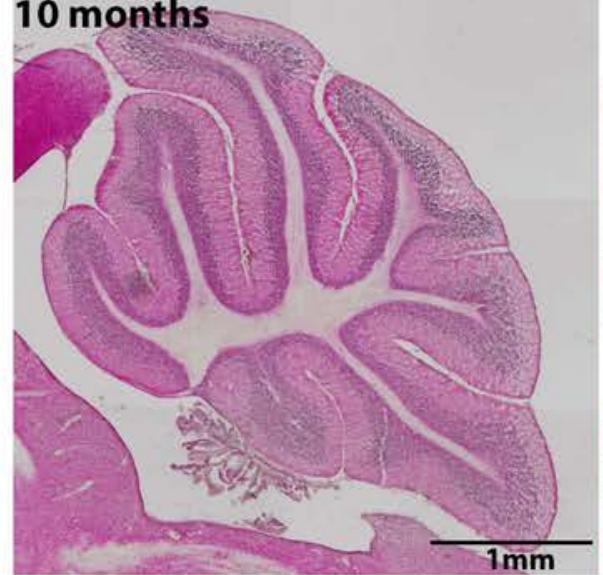

C
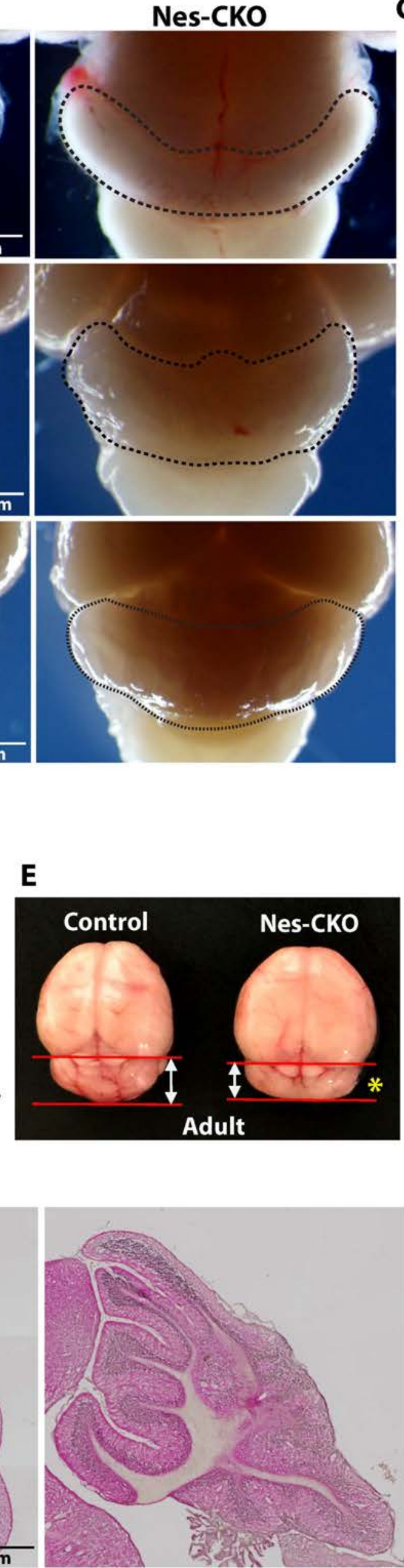

Control

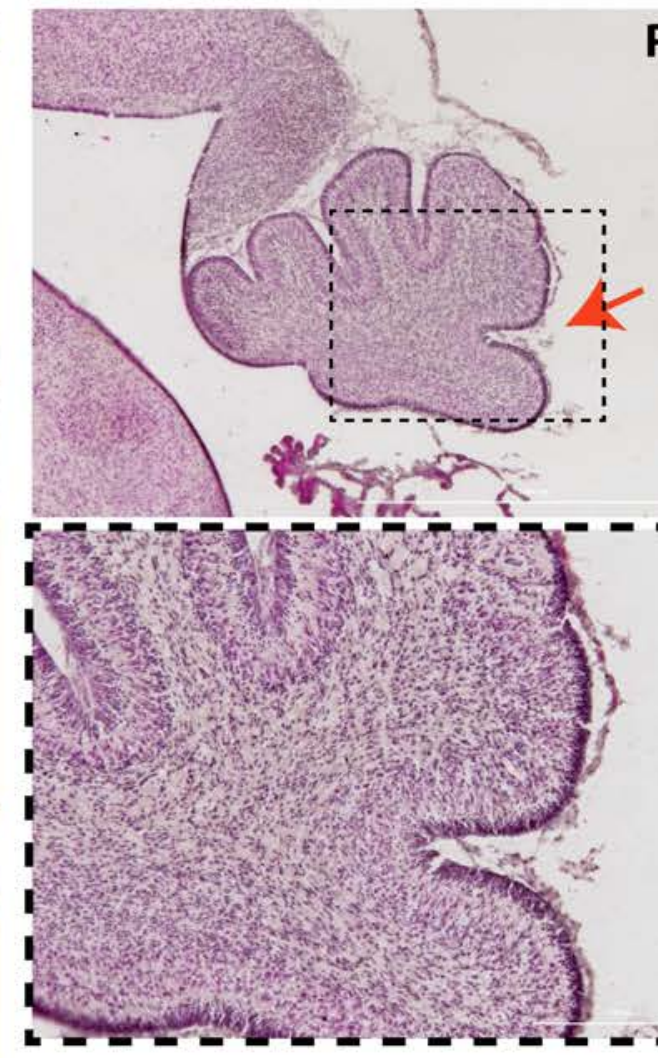

P1

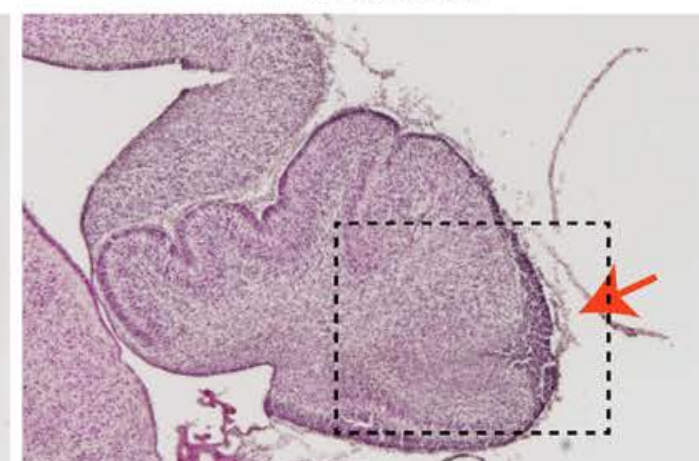

D

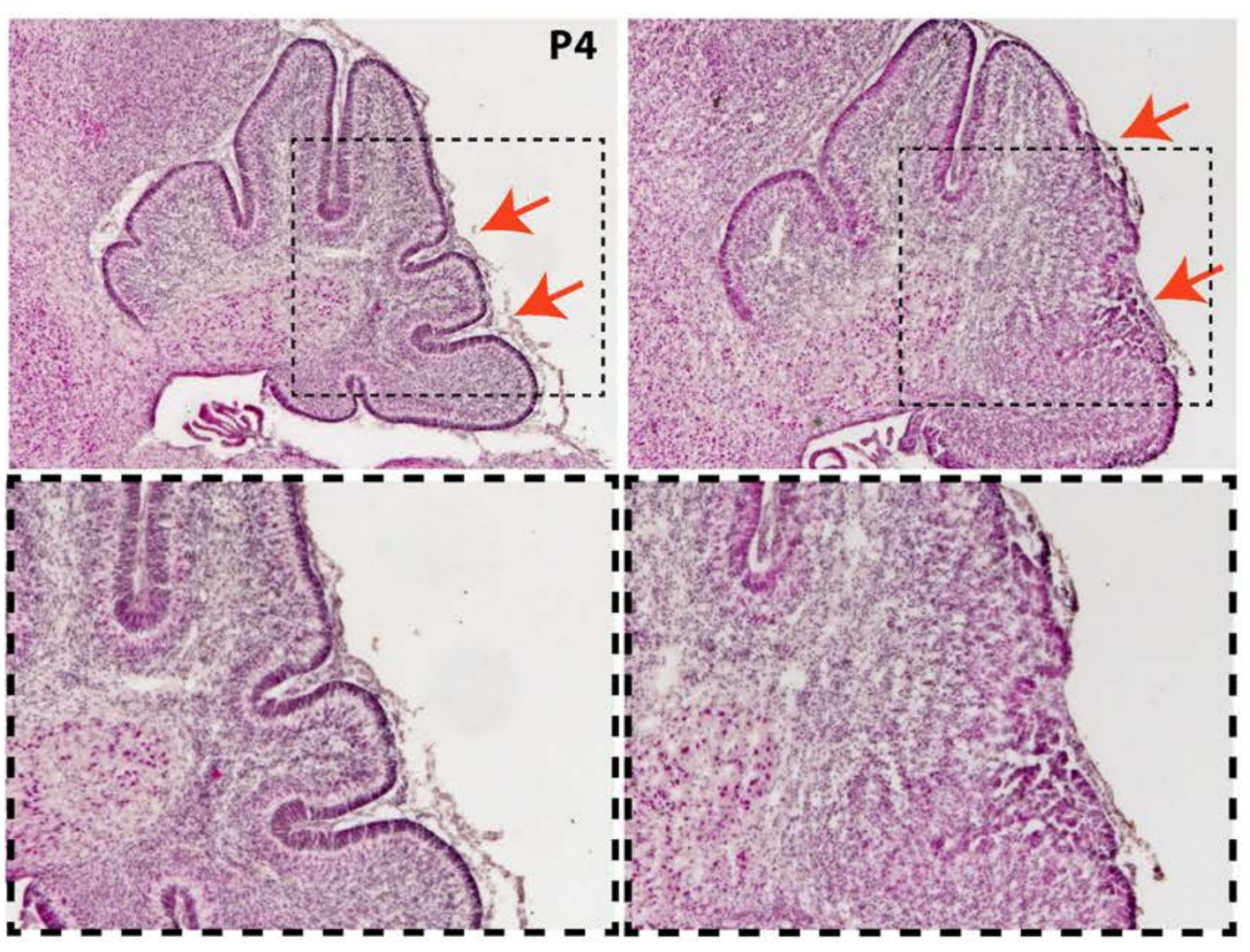


Figure 2
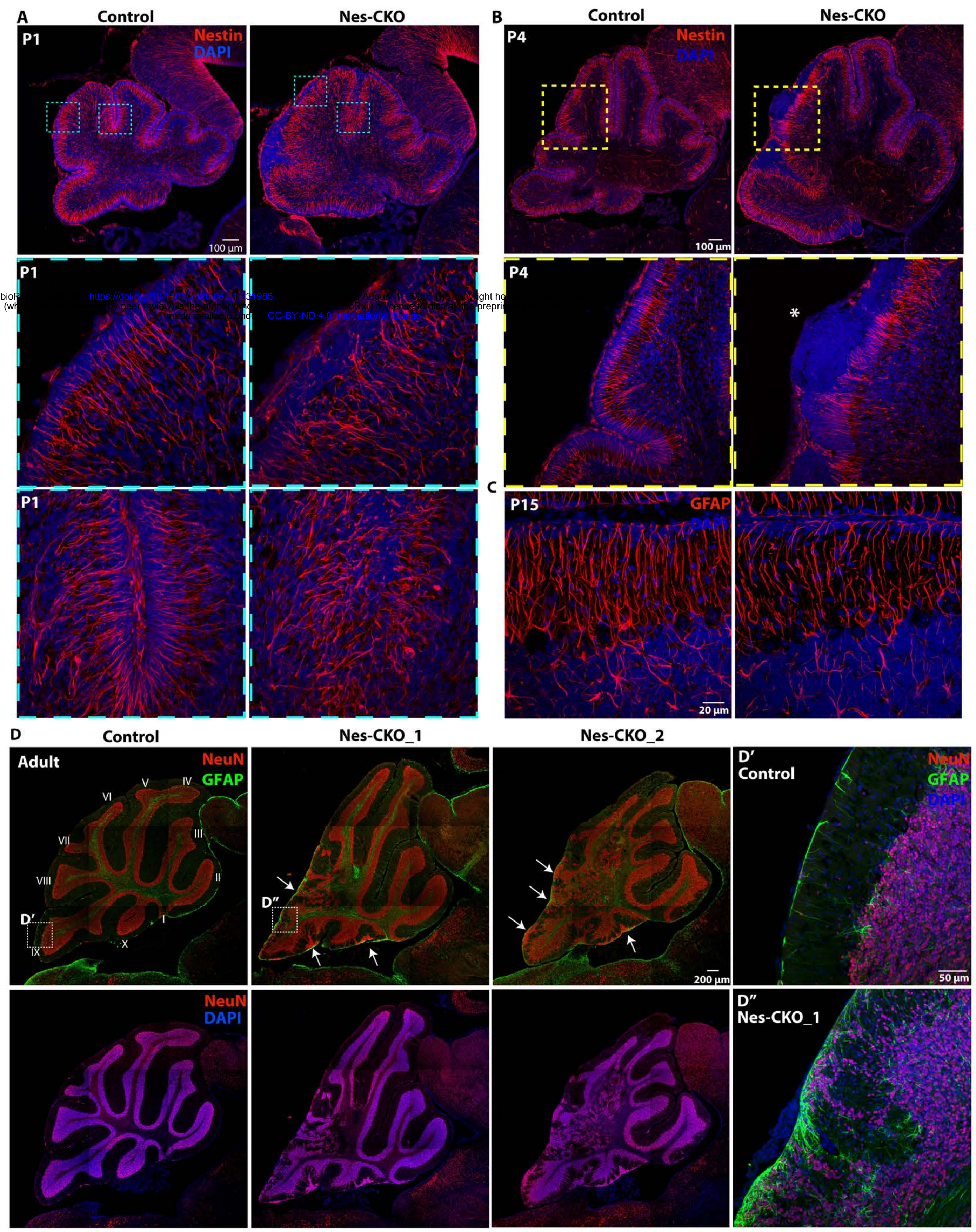
Figure 3

A

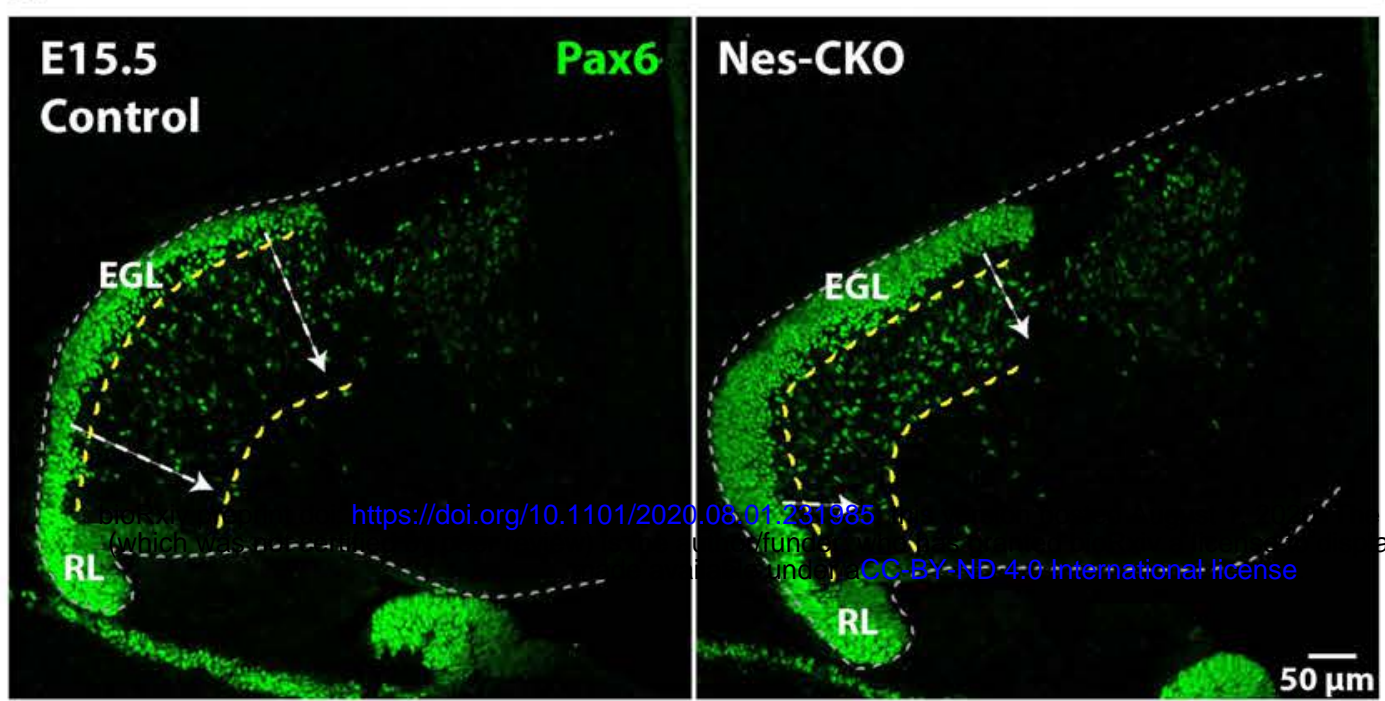

B

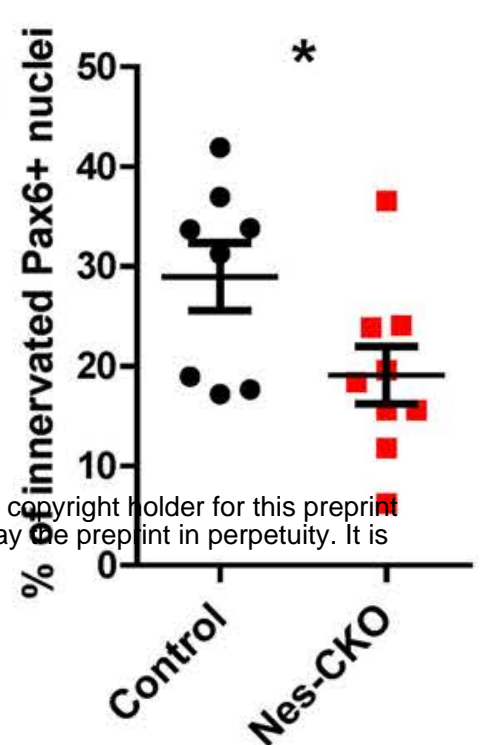

Nes-CKO

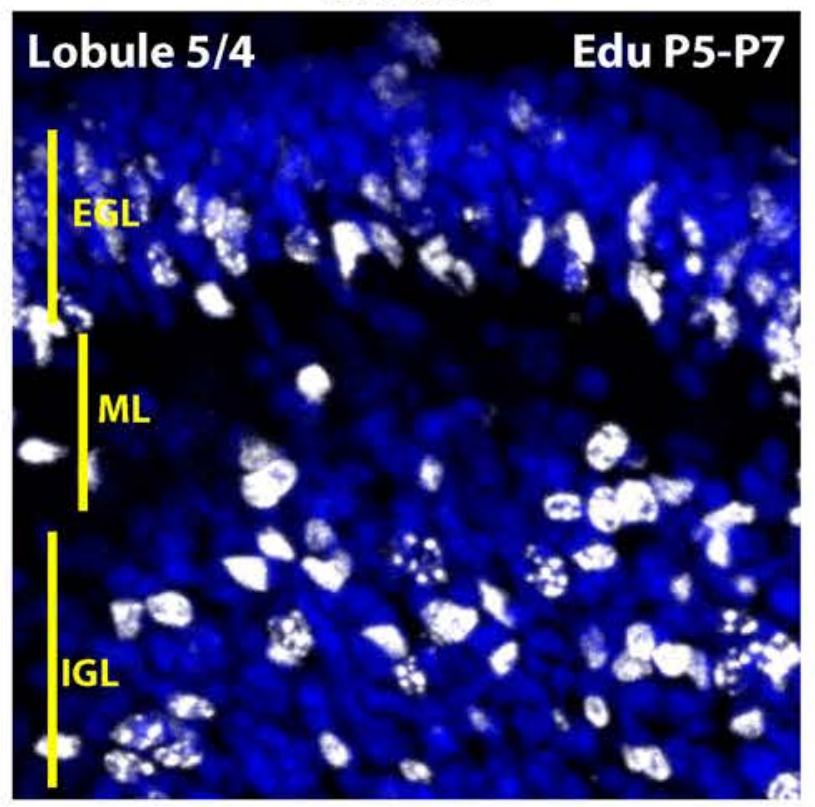

Lobule 6

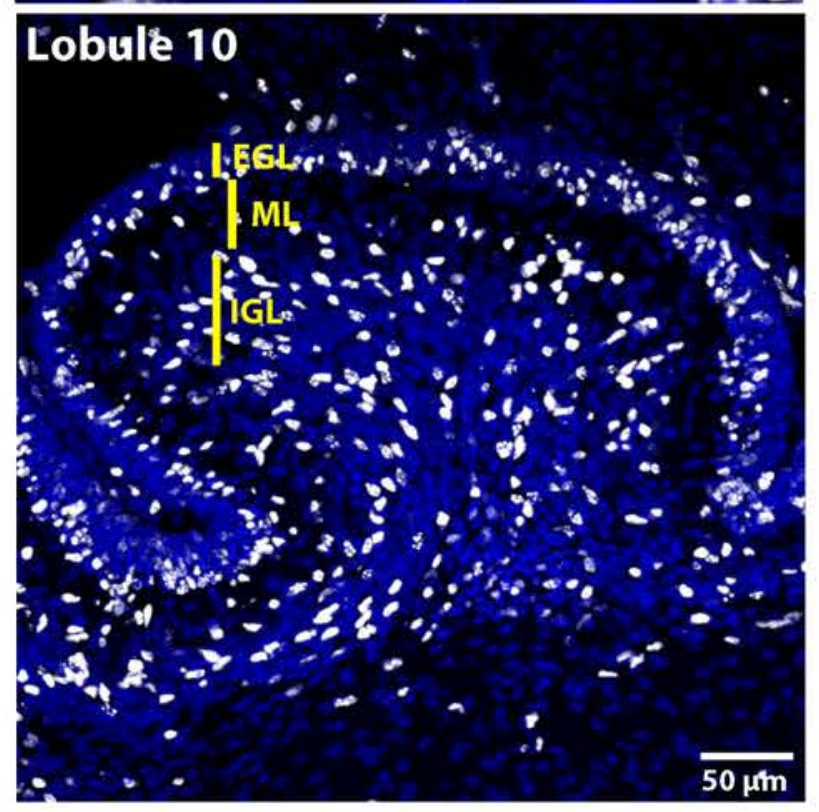

C
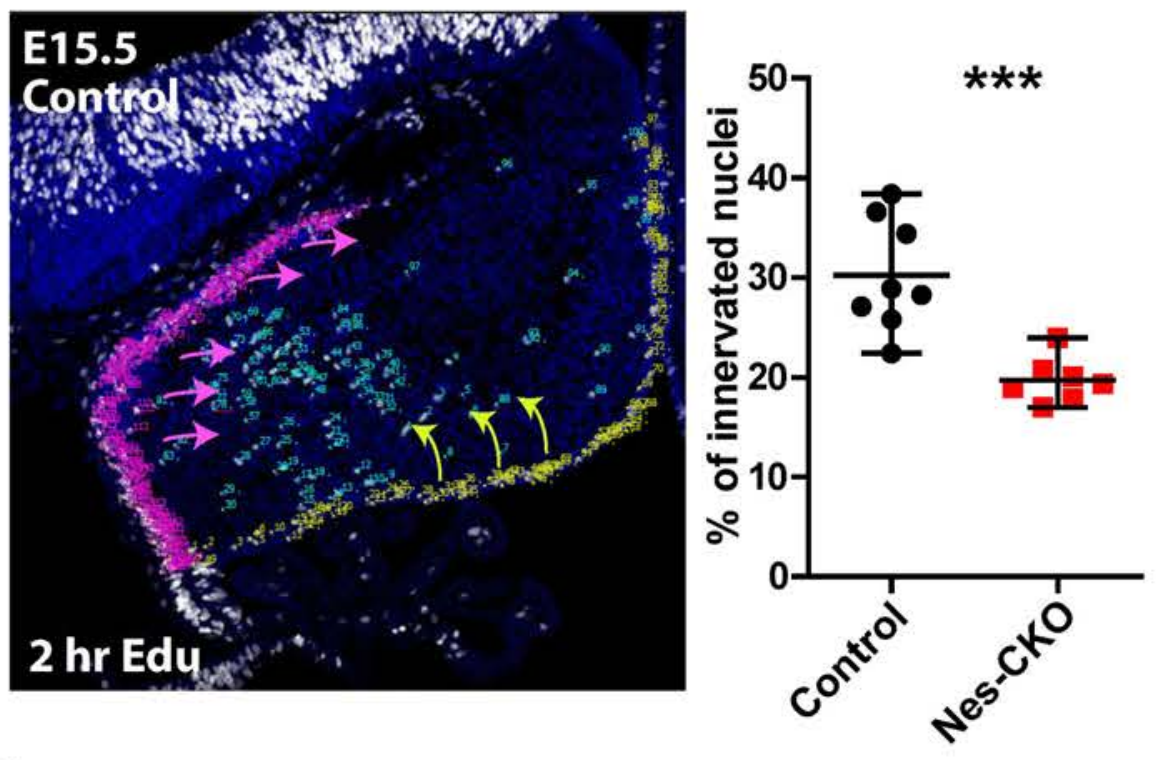

E

Lobule 5-4

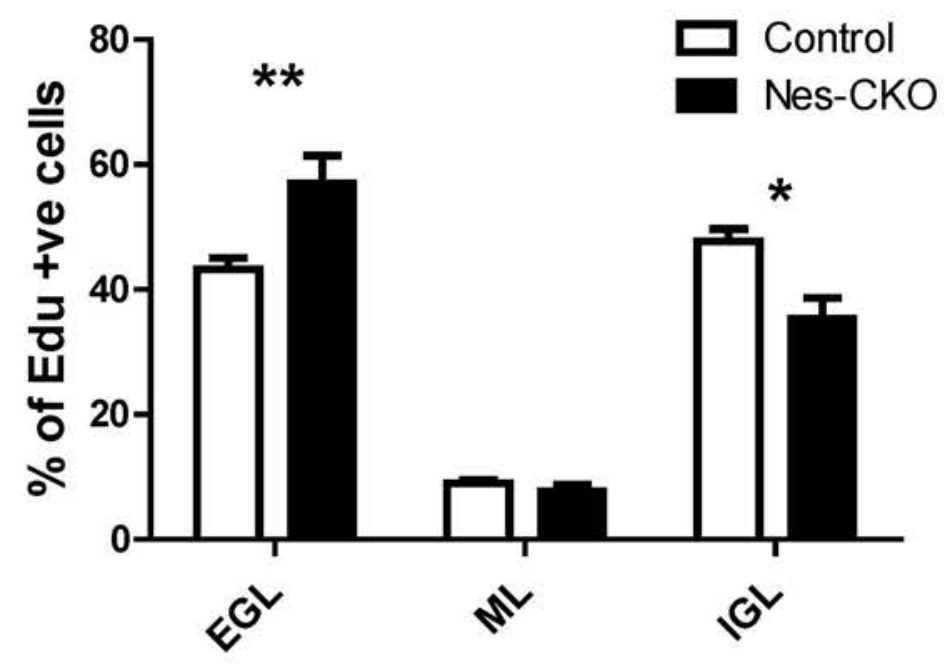

Lobule 6

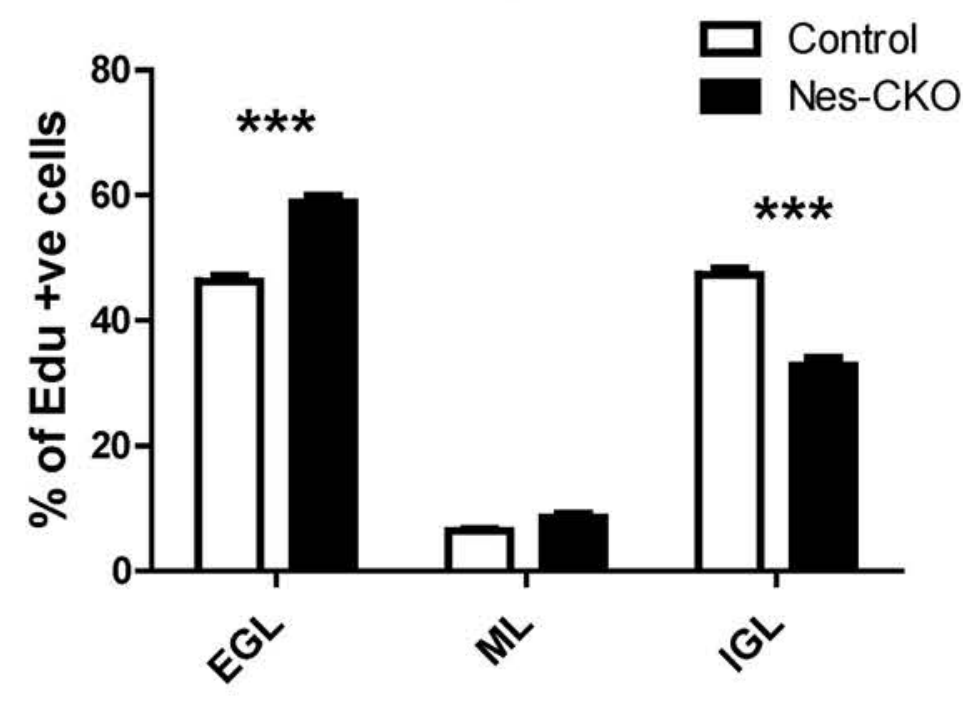

Lobule 10

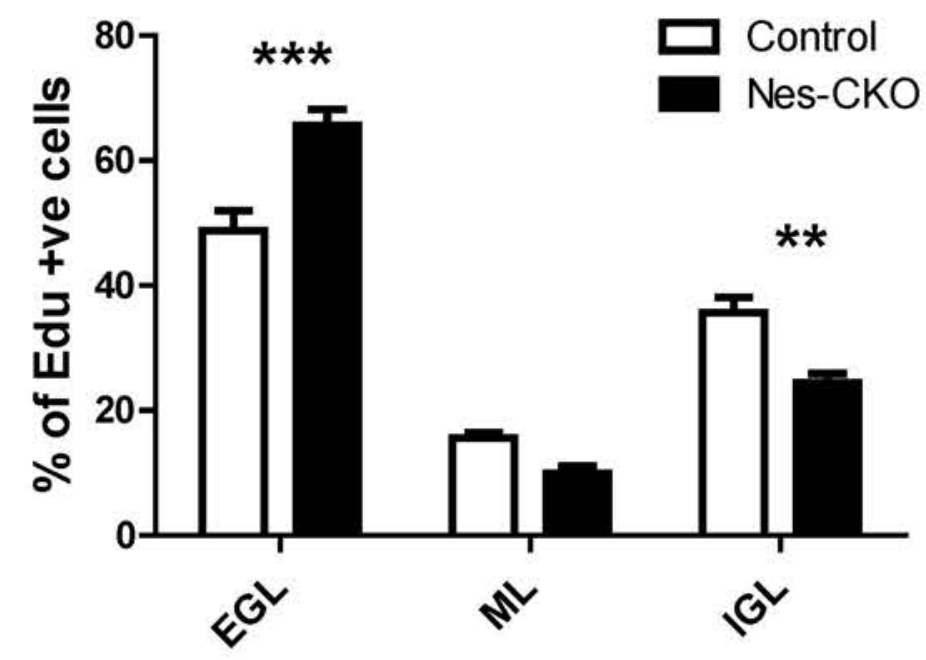




\section{Figure 4}

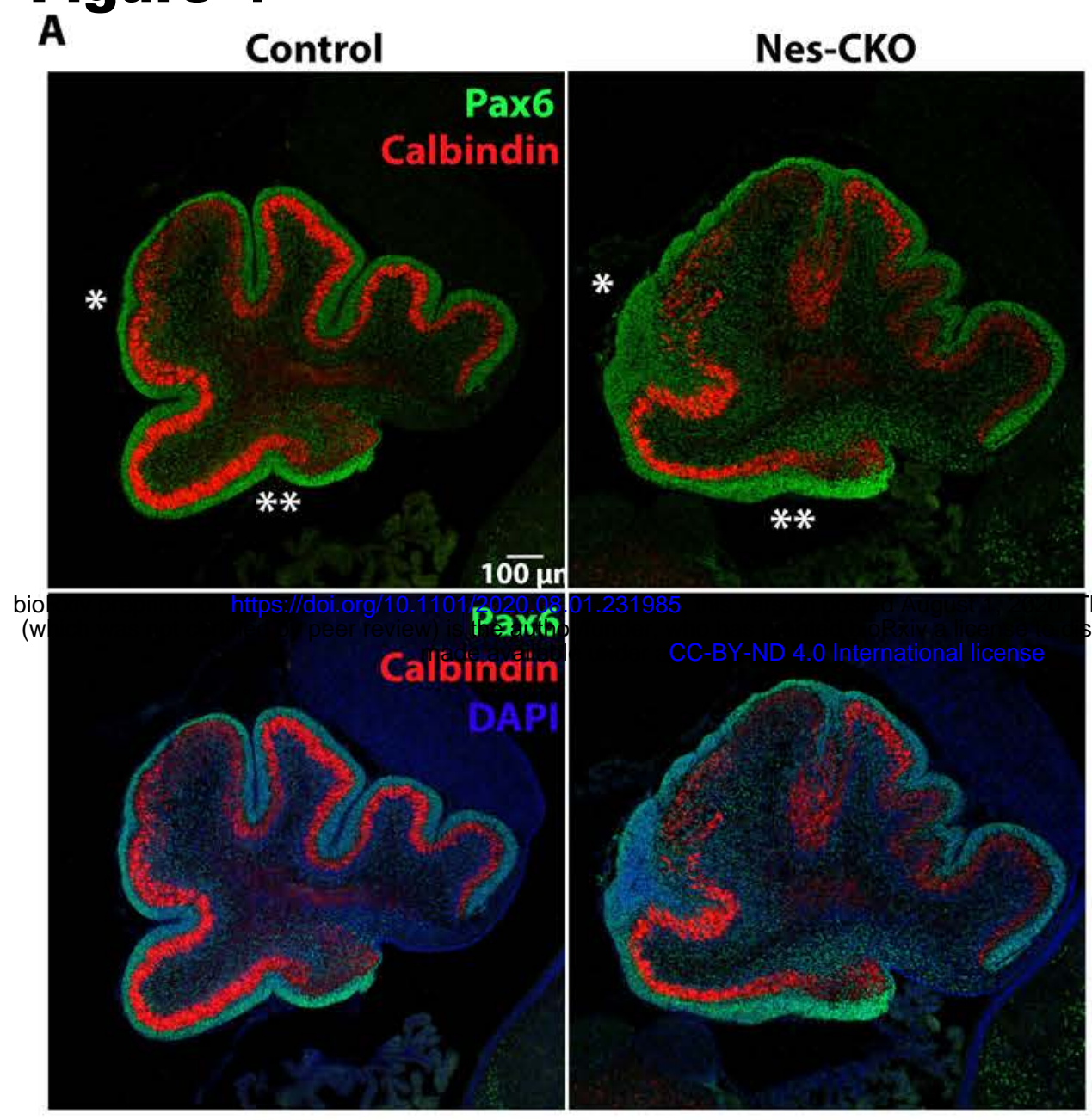

B

Control

Nes-CKO

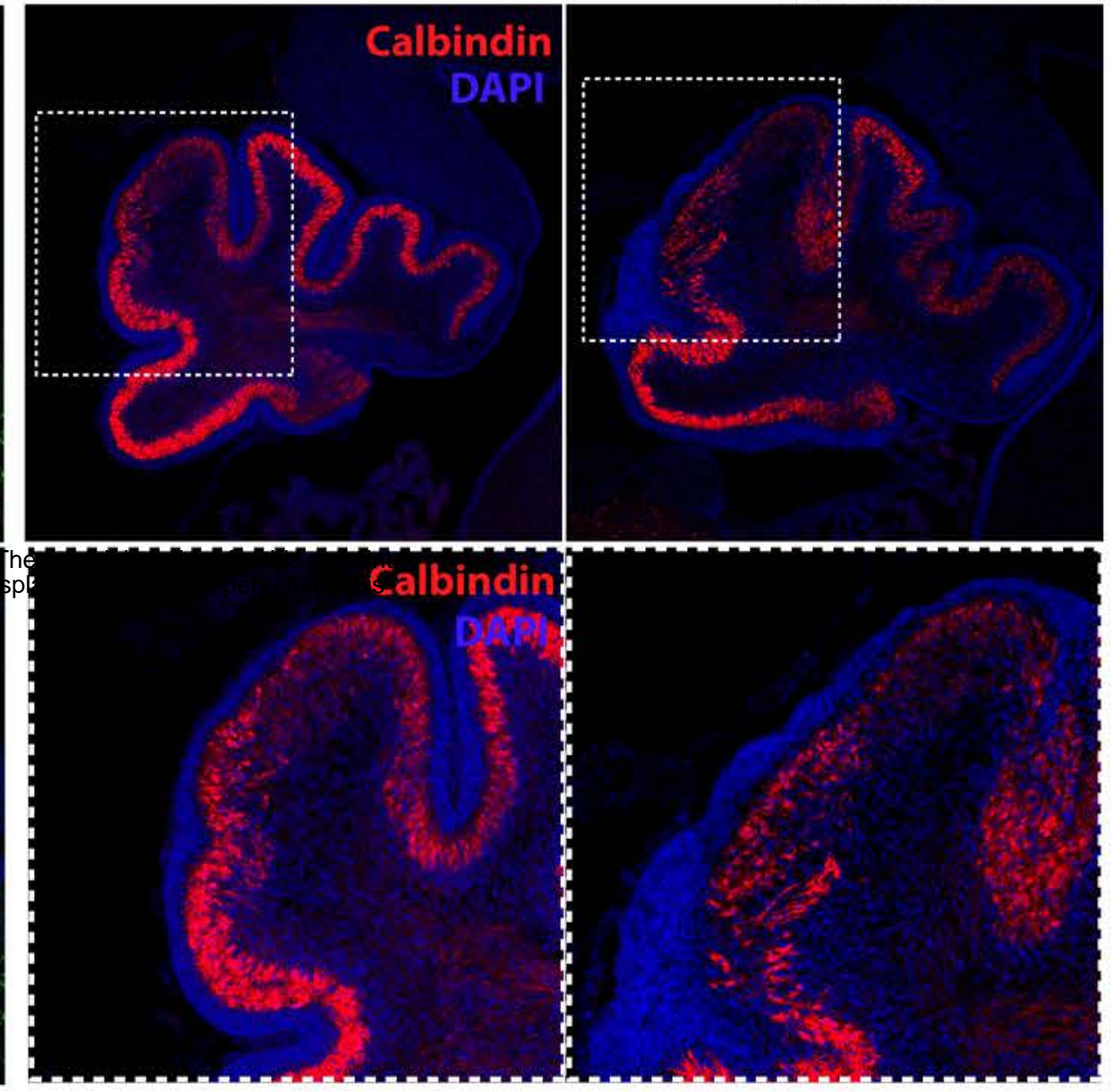

C

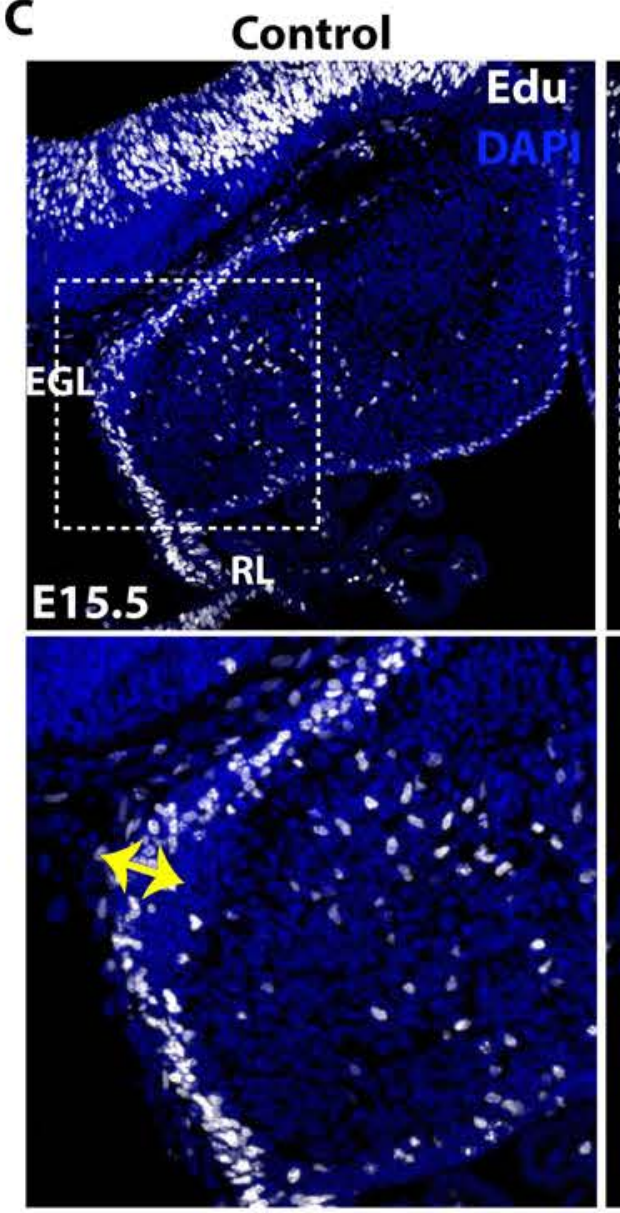

E

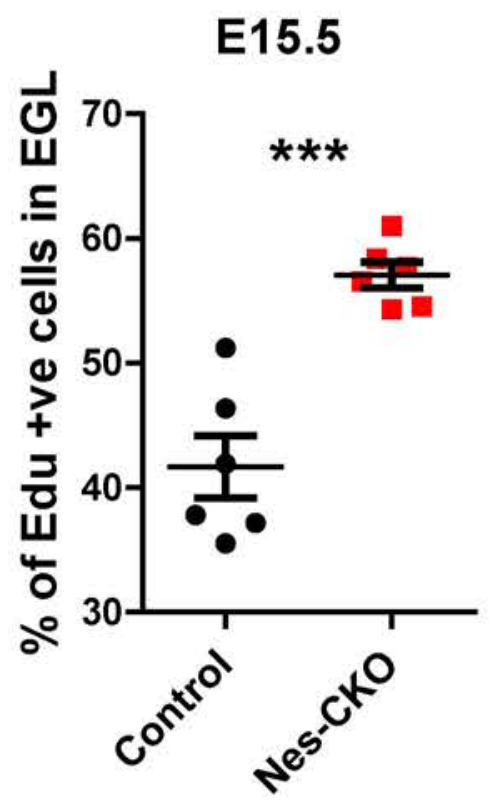

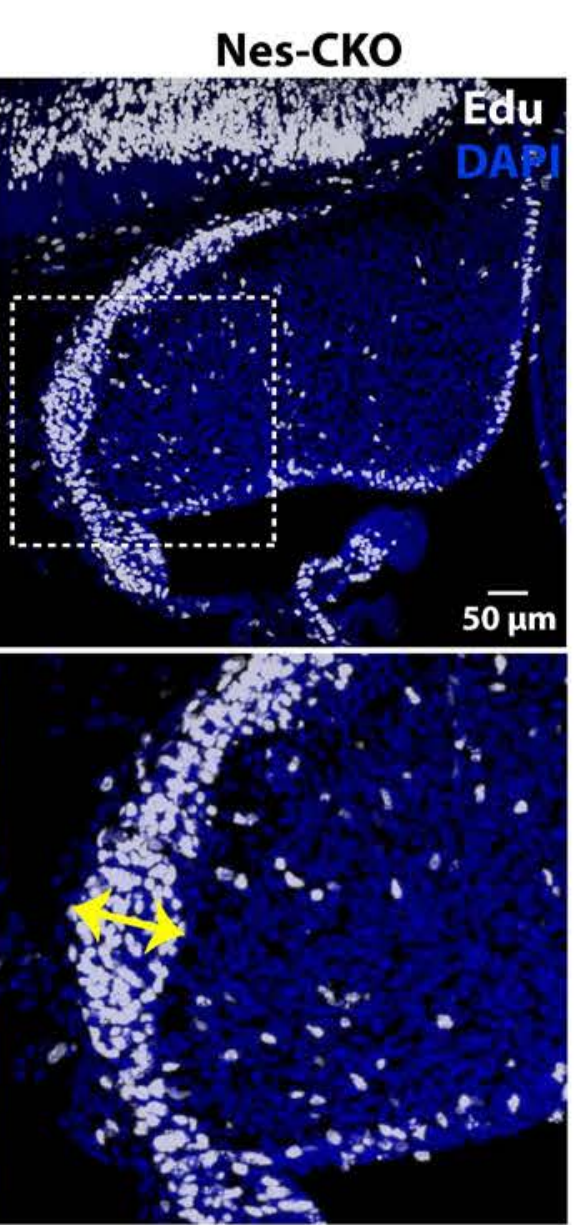

F

ki67

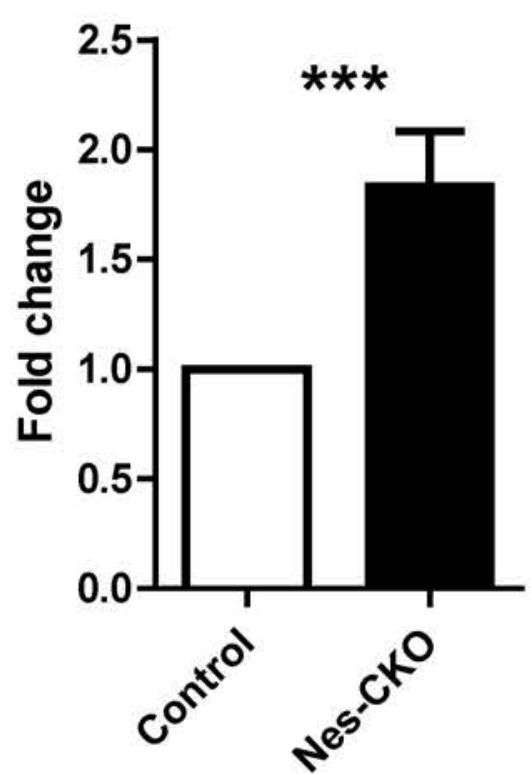

D

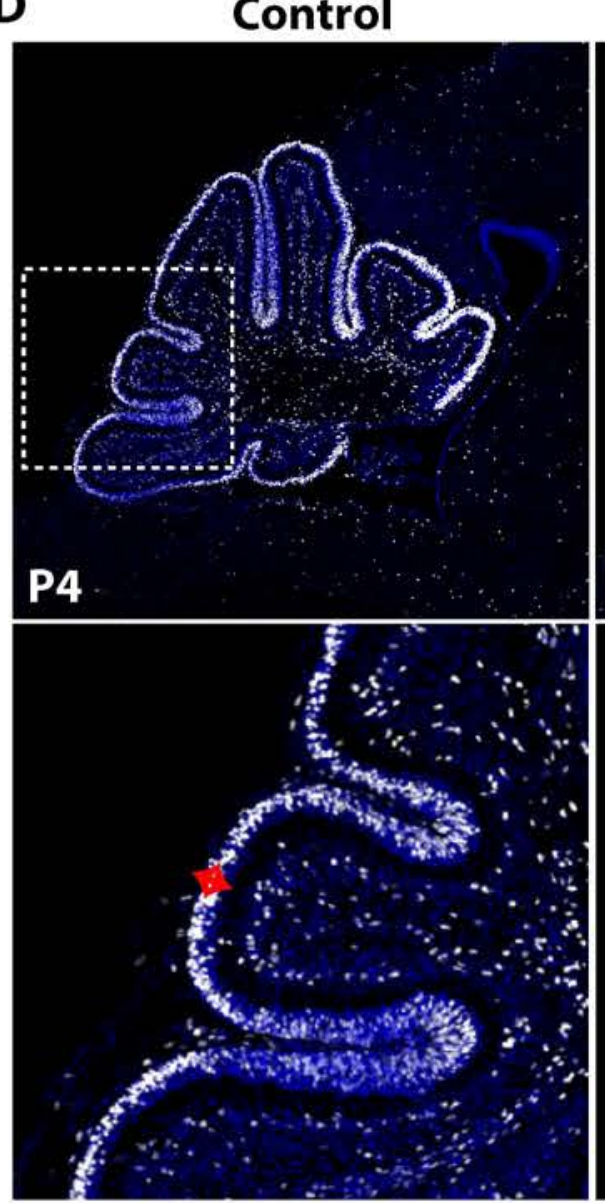

Atoh1

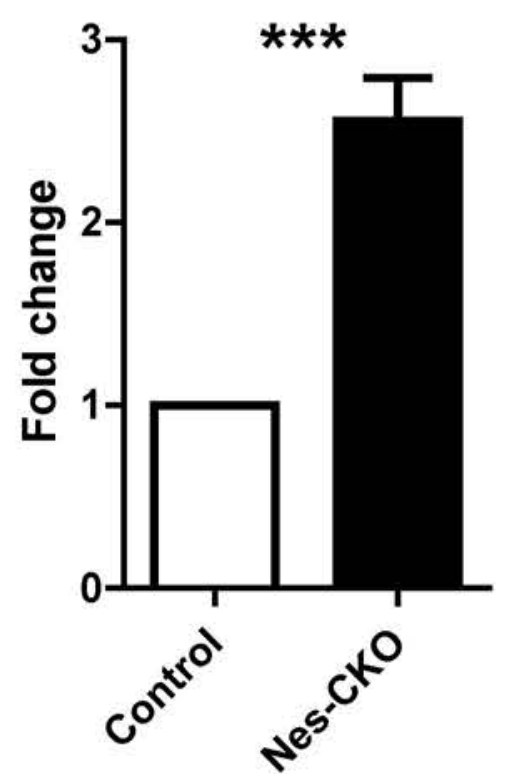

Nes-CKO

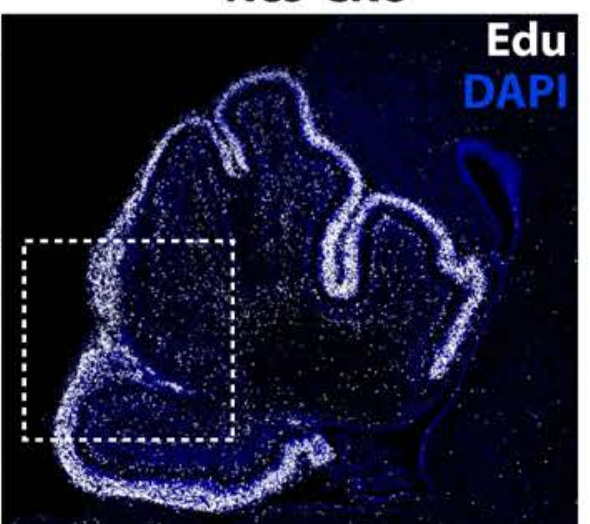

$200 \mu \mathrm{m}$

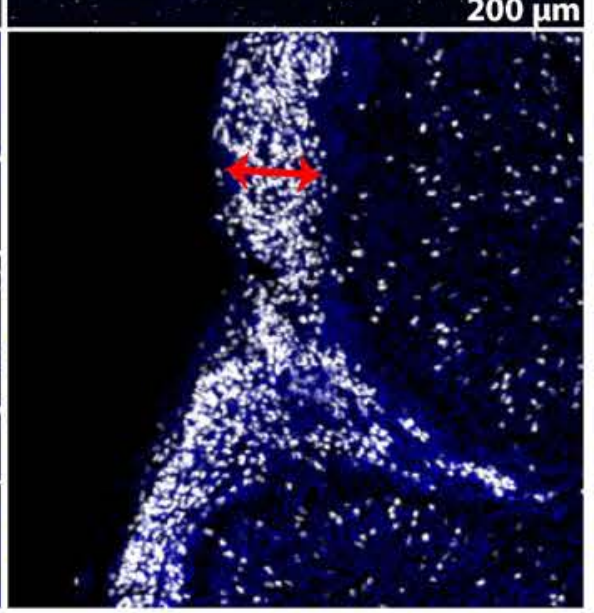

\section{Rab23}

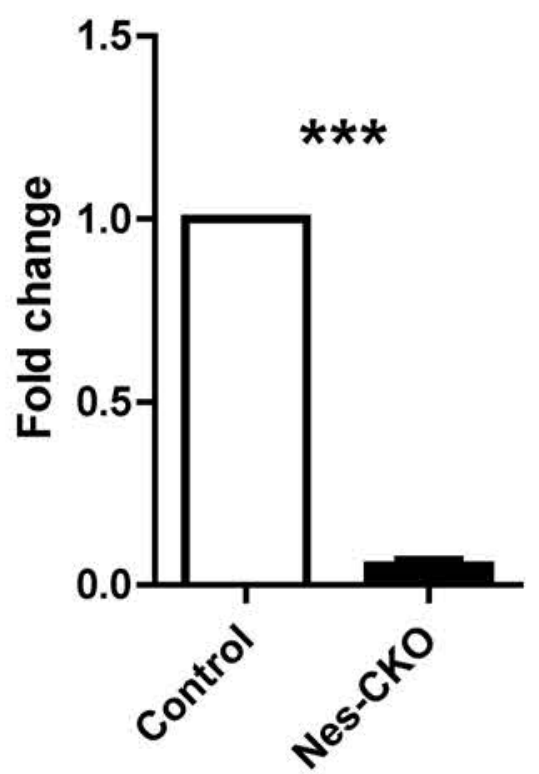


Figure 5

bioRxv preprint doi: https://doi.org/10.1101/2020.08.01.231985; this version posted August 1, 2020. The copyright holder for this preprint (which was not certified by peer review) is the author/funder, who has granted bioRxiv a license to display the preprint in perpetuity. It is

A E15.5

Gli1

Gli2

Gli3

Shh
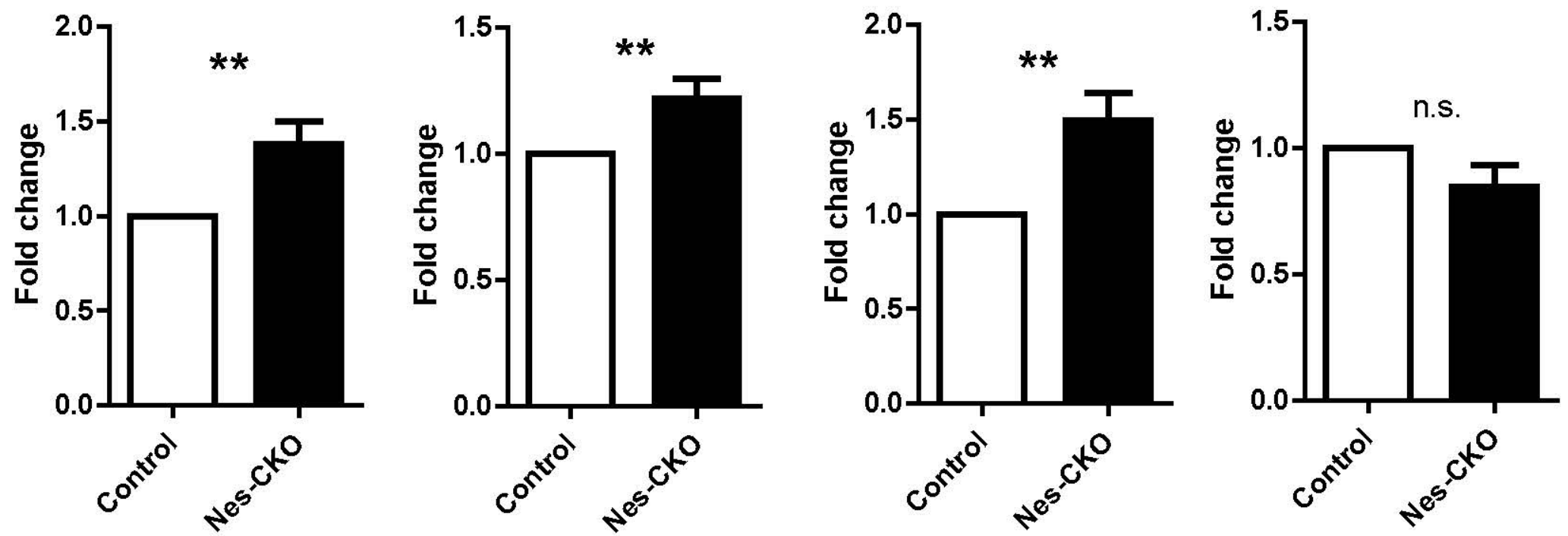

B $\mathbf{P 1 5}$
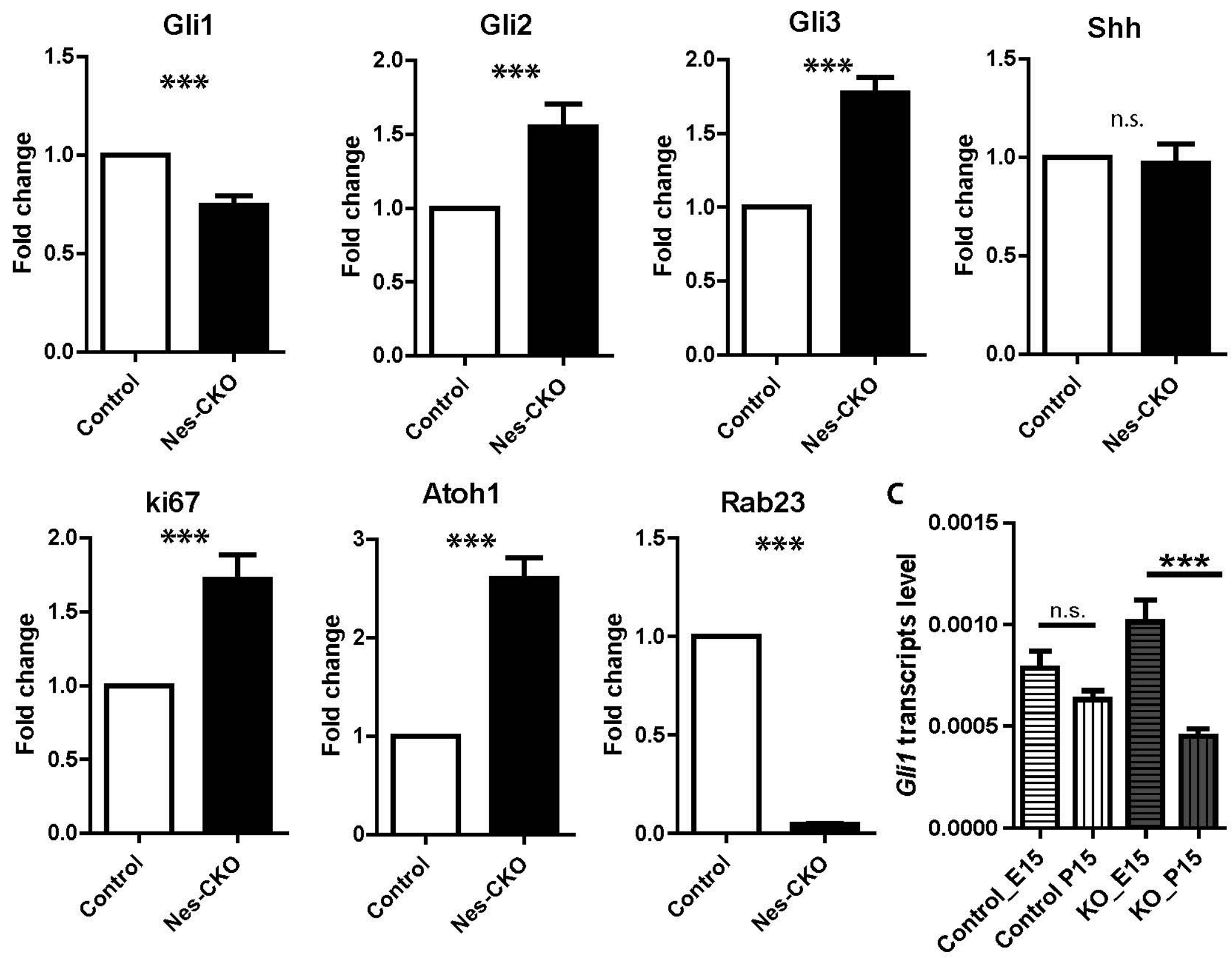


\section{Figure 6}

A
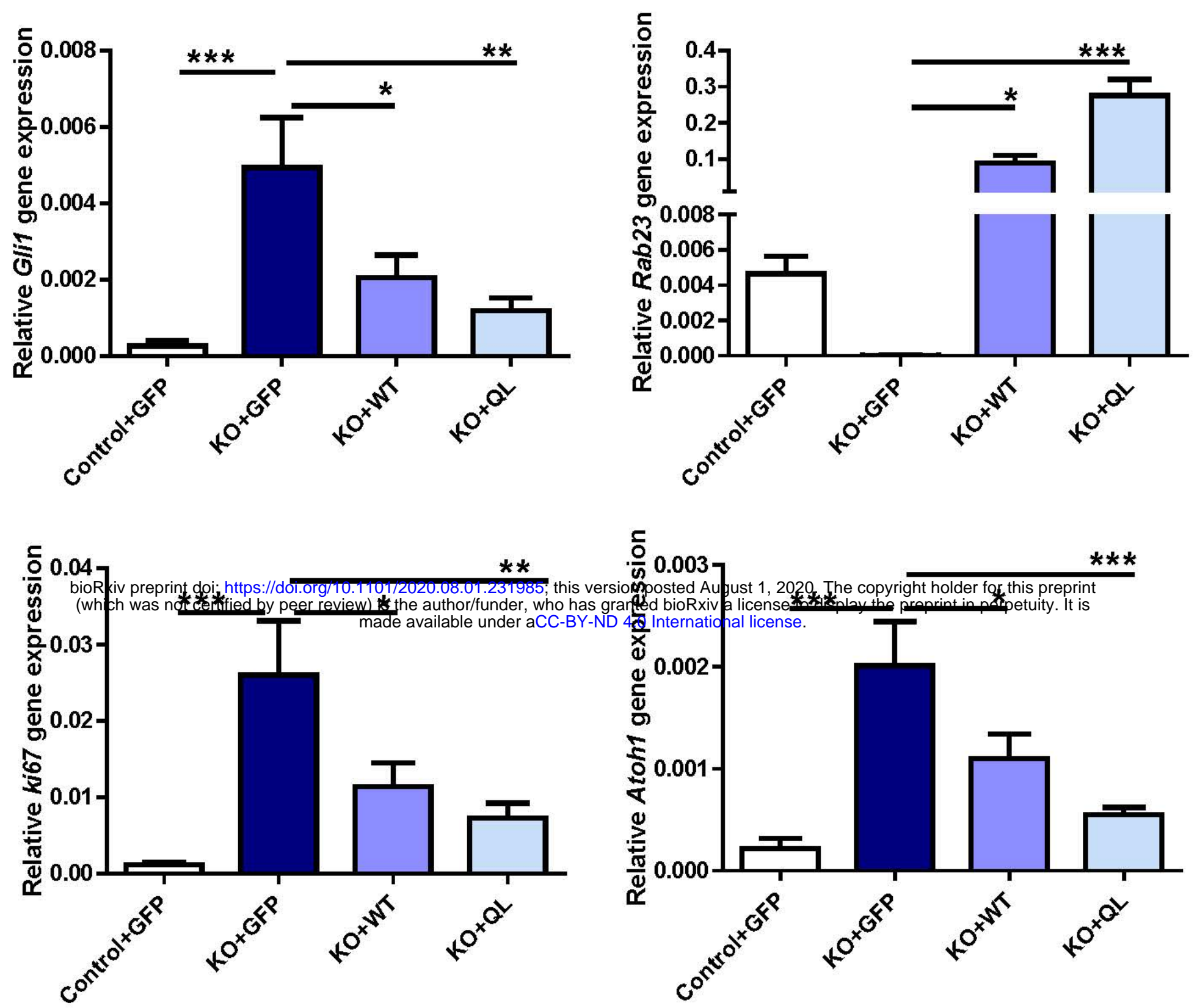

C

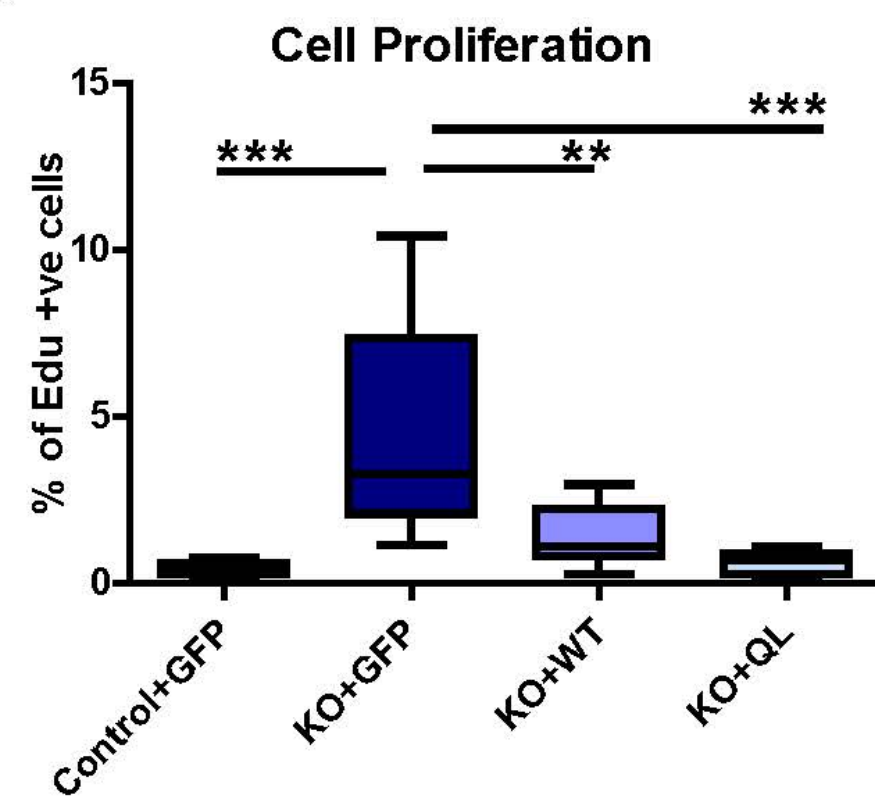

응 0.0037

moosted Au ust 1, 2020. The copyright holder fox this preprint
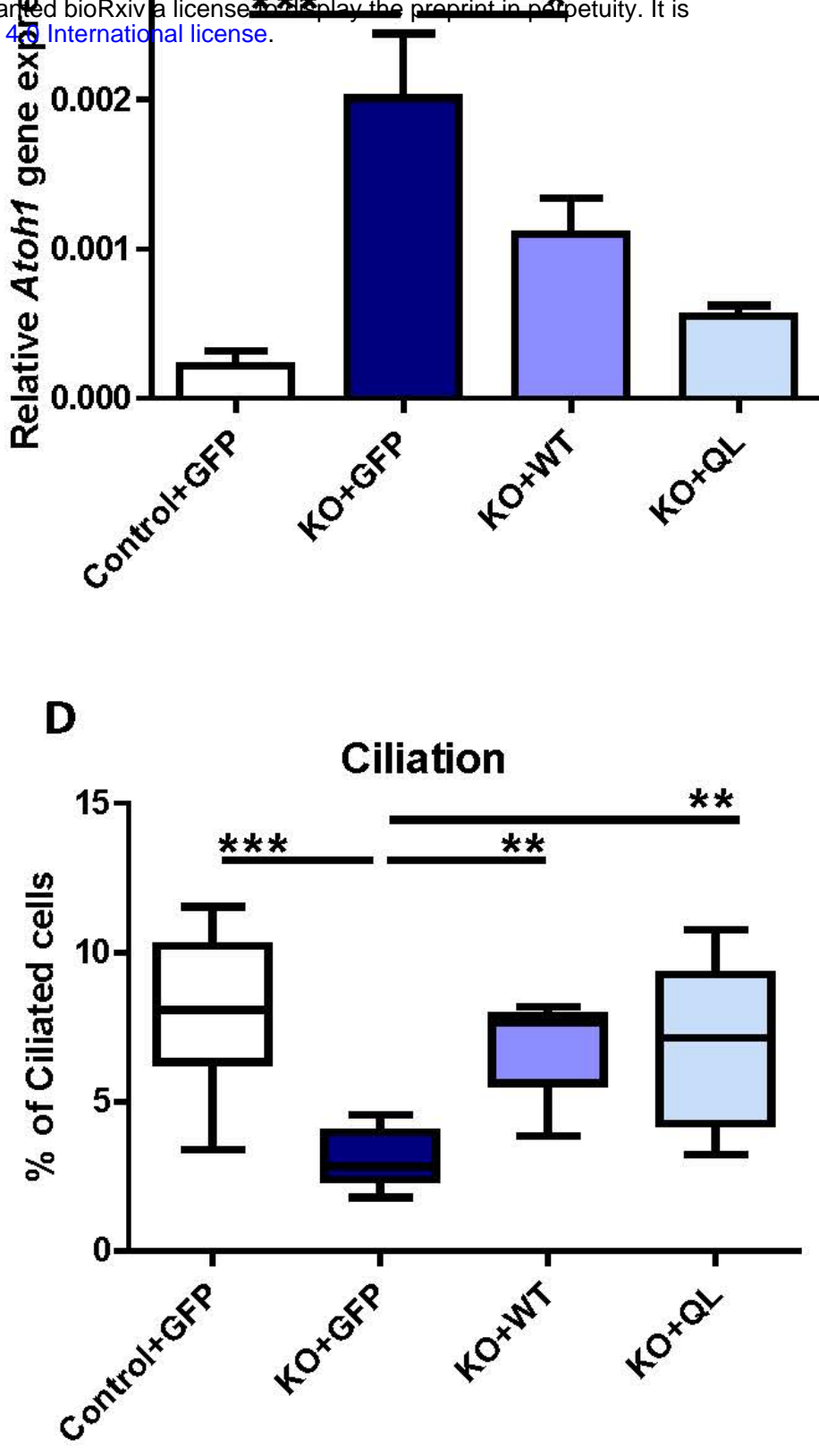
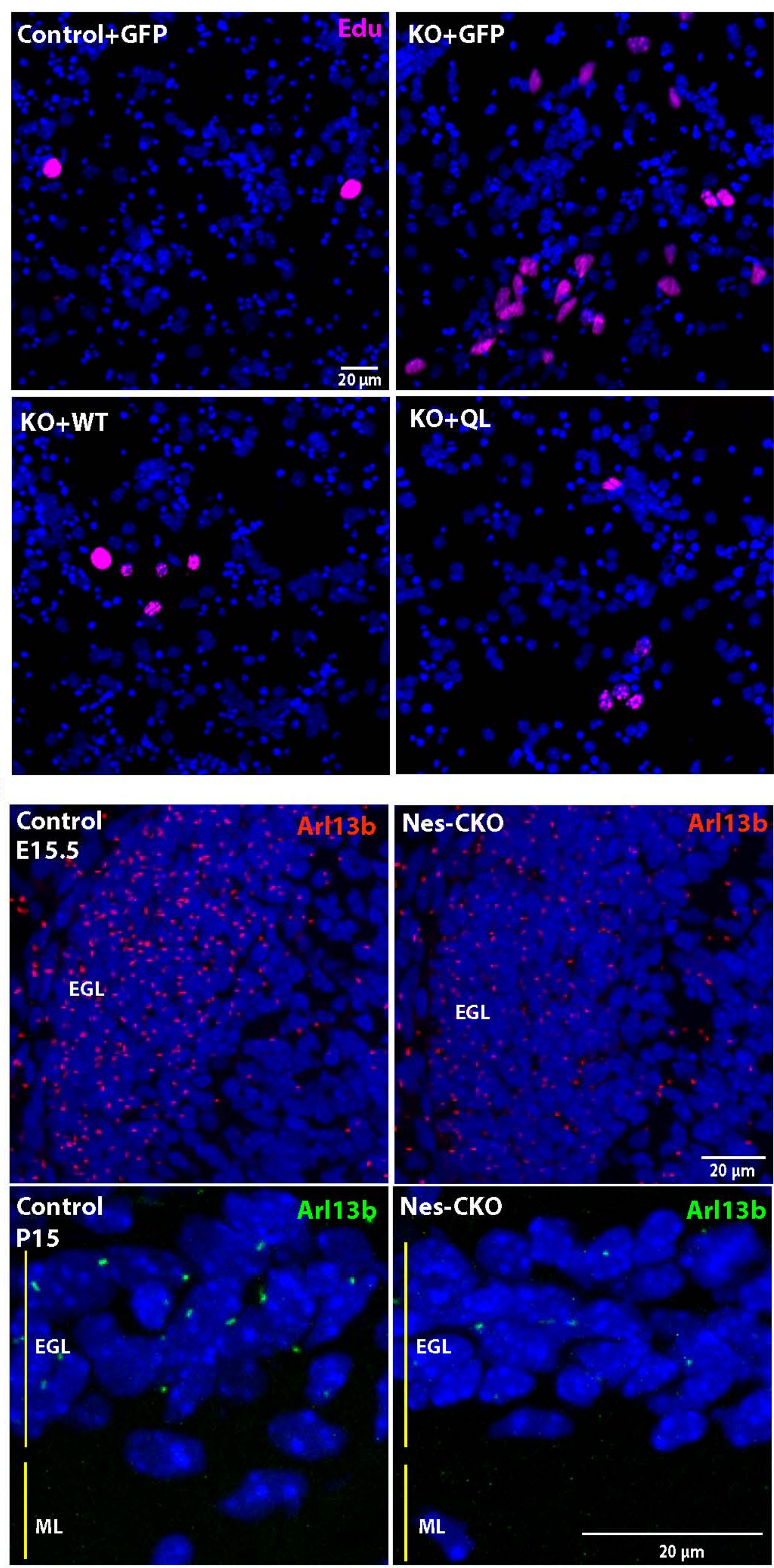

F

G
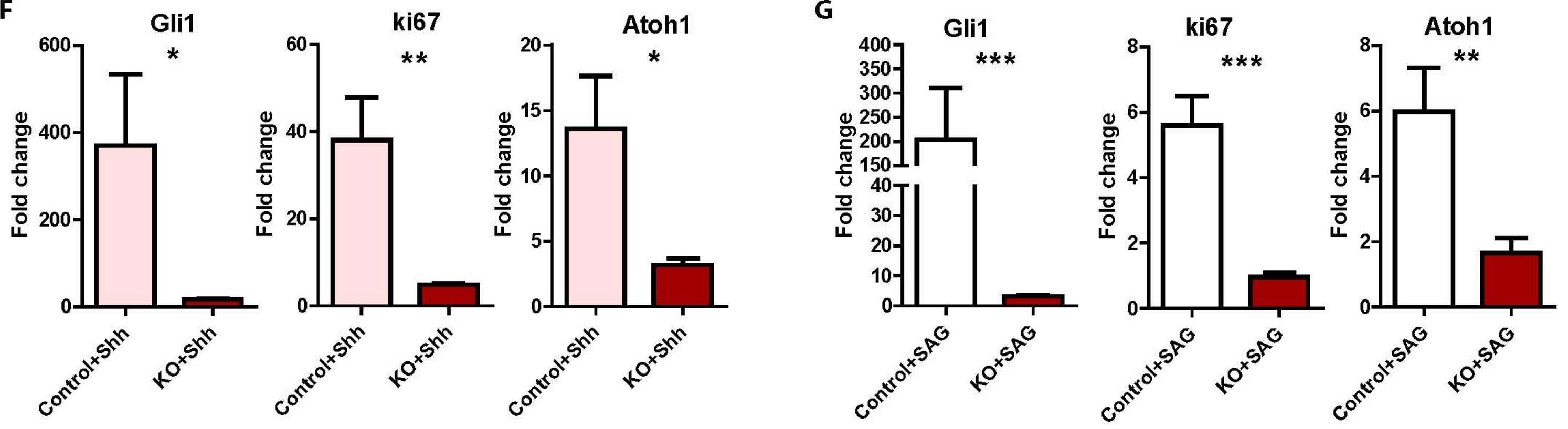
Figure 7
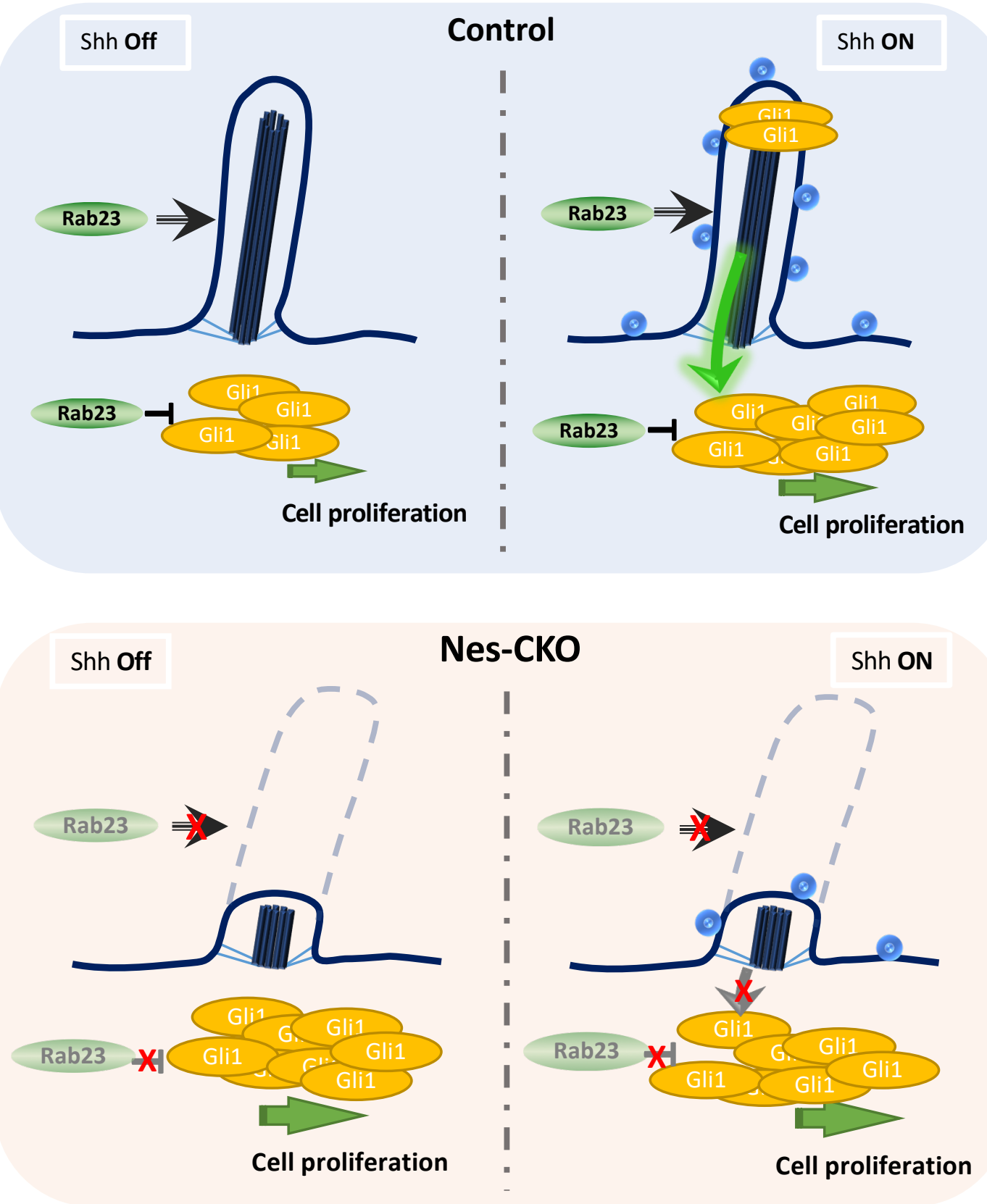